\author{
ALAIN MARCHANDISSE \\ Maître de recherches au F.N.R.S. \\ Université de Liège \\ et \\ CHRISTOPHE MASSON \\ Aspirant au F.N.R.S. \\ Université de Liège
}

\title{
LES TRIBULATIONS DU GRAND BÂTARD ANTOINE DE BOURGOGNE EN ITALIE (1475) ${ }^{1}$
}

Il est permis de s'étonner du fait que le voyage d'Italie entrepris en 1475 par Antoine de Bourgogne afin de confirmer à Ferrante d'Aragon, roi de Naples, son élévation à ce titre de chevalier de la Toison d'or qui lui avait été conféré lors du chapitre de Valenciennes de mai 1473, et de recevoir son serment juré sur les statuts de l'ordre, sujet a priori enthousiasmant et riche de potentialités, n'ait jamais fait

1 Qu'il nous soit permis de remercier chaleureusement ici tous ceux - ils sont nombreux ; nous espérons n'avoir oublié personne - qui, d'une manière ou d'une autre, ont accru la matière de la publication qui suit : Mesdames et Messieurs R. ADAM (Bibliothèque royale de Belgique, Bruxelles), A. Armstrong (University of Manchester), G. Battioni (Università Bicocca di Milano), P. Bauduin (Université de Caen), É. Bousmar (Facultés universitaires Saint-Louis, Bruxelles), J.-M. CAuchies (Académie royale de Belgique; Facultés universitaires SaintLouis, Bruxelles; Université catholique de Louvain), É. Crouzet-Pavan (Université de Paris-Sorbonne, Paris IV), A. Delfosse (FNRS/Université de Liège), G. DocQuier (Facultés universitaires Saint-Louis, Bruxelles), A. DudA (Université Charles-de-Gaulle-Lille 3), J. Dumont (Université de Liège), L. C. Gentile (Université de Chambéry; Università di Torino), O. Guyotjeannin (École des chartes, Paris), J. HAYEz (École normale supérieure, Paris), I. Lazzarini (Università degli Studi del Molise), M. Maillard-Luypaert (Grand Séminaire de Tournai ; Facultés universitaires Saint-Louis, Bruxelles), F. Neveux (Université de Caen), S. Palmieri (Istituto italiano per gli Studi storici, Napoli), G. Palumbo (Facultés universitaires Notre-Dame-de-la-Paix, Namur), A. Paravicini Bagliani (Université de Lausanne), É. Pibiri (Université de Lausanne), M. Prietzel (Humboldt-Universität zu Berlin), L. Puccio (Université de Liège), K. Rahn (Deutsches Historiches Institut in Rom), G. RicCI (Università degli Studi di Ferrara), P. SAVy (Université de Marne-la-Vallée), B. SchnerB (Université Charles-de-Gaulle-Lille 3), F. Senatore (Università degli Studi di Napoli Federico II), A.-B. SpitzBarth (Institut historique allemand, Paris ; Université Charles-deGaulle-Lille 3), J. Theurot (Université de Franche-Comté) et P. Vuillemin (École française de Rome). Comme de coutume, mon collègue et ami B. Schnerb a eu la gentillesse de relire ces quelques pages, ce dont je lui suis particulièrement reconnaissant (A.M.). Il convient 
l'objet d'une étude spécifique au sein de l'historiographie bourguignonne ${ }^{2}$. Sans doute l'enquête a-t-elle été perçue comme particulièrement périlleuse par nos devanciers et, de fait, elle s'est révélée telle pour les auteurs du présent article. Selon nous, il est au moins deux raisons complémentaires à cet état de fait. Il nous faut tout d'abord préciser que les sources bourguignonnes ne sont pas d'une grande utilité pour le sujet qui nous occupe et pour l'optique dans laquelle nous l'avons envisagé. Pour le moins étonnamment, les chroniques se montrent obstinément muettes. Aucun des auteurs qui nourrissent le quotidien de l'historien de la Bourgogne, les La Marche, les Molinet, ne semble avoir d'information à fournir sur le périple péninsulaire du Bâtard. Quant à la comptabilité, en général si riche et si précieuse pour quiconque entreprend de retracer le passé des États bourguignons, soit elle est inexistante - aucun compte annuel de l'argentier Nicolas de Gondeval n'est parvenu jusqu'à nous, par exemple-, soit elle se montre peu diserte et d'interprétation malaisée, à l'image, au sein du contrôle de l'argentier, des rôles mensuels de paiement pour le duc à l'année $1474^{3}$ - on le verra plus tard lorsqu'il sera question du départ d'Antoine ${ }^{4}$. Seconde raison, sans doute, à la désaffection que nous pointions du doigt tout à l'heure, à propos du thème qui nous intéresse ici : les efforts que nécessite la reconstitution des tribulations en terre italienne d'Antoine de Bourgogne, dans ses pas en quelque sorte, ceux que se doit de consentir le médiéviste soucieux d'épuiser le sujet choisi, en d'autres

enfin de signaler qu'une version antérieure de ce texte a fait l'objet d'une communication à l'Université de Ferrare, le 3 décembre 2008, à l'invitation du Prof. Dr G. Ricci. Elle a pu être nuancée grâce aux remarques qu'elle a suscitées parmi l'assistance. Nous lui en sommes tout particulièrement reconnaissants. Sigles utilisés : ASMi = Archivio di Stato di Milano. - ASTo = Archivio di Stato di Torino. $-D B I=$ Dizionario biografico degli Italiani. $L D M=$ Lexikon des Mittelalters. Pour la cohésion de notre propos, les noms des intervenants transalpins ont été mentionnés sous leur forme italienne ; les toponymes se présentent sous leur forme française, s'il y échet.

2 R. Vaughan, Charles the Bold, rééd., Woodbridge, 2002, pp. 236-237. - R. J. WaLsh, Charles the Bold and Italy (1467-1477). Politics and Personnel, Liverpool, 2005, pp. 143, 219, 256257, 286, 300, 345. - ID., Relations between Milan and Burgundy in the period 1450-1476, dans Gli Sforza a Milano e in Lombardia e i loro rapporti con gli stati italiani ed europei (1450-1535). Convegno internazionale, Milano, 18-21 maggio 1475, Milan, 1982, p. 382.A. Commies, Nul ne s'y frotte. Een biografische schets van Anton, bastaard van Bourgondie, dans Excursiones mediaevales. Opstellen aangeboden aan Prof. Dr. A. G. Jongkees door zijn leerlingen, Groningue, 1979, p. 69. - J. Clément, Antoine de Bourgogne, dit le Grand Bâtard, dans La justice dans les États bourguignons et les régions voisines aux XIV-XVI siècles : institutions, procédure, mentalité, éd. J.-M. CAuchies, Publication du Centre européen d'Études bourguignonnes, t. 30, Rencontres de Luxembourg (28 septembre au $1^{\text {er }}$ octobre 1989), 1990, pp. 176-177 (ou dans Bulletin du Cercle d'Histoire et d'Archéologie Segnia, t. 26, 2001, pp. 5-19). - F. Jeuris, Antoine Grand Bâtard de Bourgogne, Mém. de Lic. en Histoire dactyl., Université de Liège, 2000-2001, pp. 135-137.

3 Cf. R.-H. BAutier, J. Sornay, Les sources de l'histoire économique et sociale du Moyen Âge, t. 2, Les États de la Maison de Bourgogne, vol. 1, Archives centrales de l'État bourguignon (1384-1500). Archives des principautés territoriales, 1. Les principautés du Sud, 2. Les principautés du Nord (supplément), Paris, 2001, p. 45.

4 Voir n. 27. 
termes la visite des dépôts d'archives et bibliothèques de l'ensemble des villes dans lesquelles Antoine fit étape durant son circuit. Compte tenu de notre souci d'exhaustivité, même s'il ne nous a pas été possible de consacrer des mois à une sorte de Giro heuristique ${ }^{5}$ et si la bibliographie italienne, sources et travaux, se révèle souvent bien clairsemée dans les bibliothèques belges, c'est à une recherche aussi complète, précise et circonstanciée que possible, enrichie, il est vrai, nous avons plaisir à le souligner, par les collègues et amis étrangers spécialistes d'histoire italienne, que nous convions le lecteur. À cet égard, nous suivrons deux grands axes. Nous allons tout d'abord tenter de retracer, dans la mesure du possible, l'itinéraire suivi par le Bâtard et d'évoquer chacune des cités où il fut reçu, l'accueil qui lui fut réservé, les activités qu'il y mena. Dans un second temps, il s'agira de s'interroger sur les raisons qui conduisirent Antoine à s'engager dans ce périple italien. Il y en a selon nous plus d'une et ces divers motifs nous semblent tenir, pour l'essentiel, à la situation que connaissaient alors les États du duc Charles le Hardi. Mais commençons tout d'abord par feuilleter un carnet de voyage ou tout au moins ce qu'il nous a été possible d'en reconstituer, au départ de dizaines de témoignages épars, parmi lesquels les plus riches sont assurément les dépêches des ambassadeurs, milanais ou ayant séjourné à la cour du duc Sforza ${ }^{6}$.

Dans le courant de février $1475^{7}$ - la date ne paraît pas pouvoir être affinée -, Antoine de Bourgogne, demi-frère du Téméraire ${ }^{8}$, dont l'on a dit, manifestement à tort, que l'appellation de « Grand Bâtard » dont il se voit généralement doté dans

5 Tout en n'ayant pas pu - la régularité de la parution des Publications du Centre est de bon aloi, mais aussi assez impitoyable -, ni su sans doute parfaitement leur donner le traitement exhaustif qu'ils méritaient, persuadés par ailleurs qu'il en reste de nombreux à découvrir, nous nous sommes efforcés de tirer le meilleur parti possible de la masse de documents découverts dans les archives italiennes. Nous nous proposons d'en éditer les pièces majeures dans une publication ultérieure, laquelle nous permettra par ailleurs d'accroître, de nuancer voire de corriger le présent propos.

6 L'on aura une bonne idée de l'apport potentiel de ces sources dans G. Soldi-RondinINI, Aspects de la vie des cours de France et de Bourgogne par les dépêches des ambassadeurs milanais (seconde moitié du XV siècle), dans Adelige Sachkultur des Spätmittelaters. Internationaler Kongress Krems an der Donau 22. bis 25. September 1980, Vienne, 1982, pp. 195-214.

7 Le 7 février 1475, Galeazzo Maria Sforza, duc de Milan, déclare à Antoine que, s'il décide de venir en Italie, il l'invite à passer par le duché de Milan. À une date située après le 18 février et avant le 23, Giovanni Pietro Panigarola, ambassadeur du duc de Milan auprès de Charles le Téméraire, informe son maître qu'il a rencontré le Bâtard. Voir Carteggi diplomatici fra Milano sforzesca e la Borgogna, t. 1, 8 Marzo 1453-12 Luglio 1475, éd. E. Sestan, Rome, 1985, pp. 404-405, 406-409.

8 Antoine (*c. 1428-† 1504), comte de la Roche, de Sainte-Menehould, de Guînes, seigneur de Crèvecoeur et de Beveren(-Waas), chevalier de la Toison d'or (1456), fils de Philippe le Bon et de Jeanne de Presles (ou Prelle), époux de Jeanne-Marie de La Viefville et père de quatre enfants, dont Philippe, seigneur de Beveren, fut principalement homme de guerre et ambassadeur au service des ducs Philippe (six ambassades entre 1457 et 1466, cf. la base de données de Mme A.-B. SpItzBARTH, http://ambassadeurs.plb.free.fr/ [accès limité], 
la littérature, est controuvée par les sources bourguignonnes ${ }^{9}$ alors qu'elle apparaît, non seulement chez Philippe de Commynes ${ }^{10}$, dans la Chronique scandaleuse de Jean de Roye ${ }^{11}$ et chez certains chroniqueurs italiens ${ }^{12}$, mais aussi sous la plume de

fondement de sa thèse de doctorat sur ambassades et ambassadeurs sous Philippe le Bon, à paraître) et Charles, avant d'être fait prisonnier à Nancy et de rallier la cause du roi de France Louis XI. À son propos, voir J.-M. CAuchies, Art. Antoine de Bourgogne, dans Les Chevaliers de l'Ordre de la Toison d'or, sous la dir. de R. De SMedt, $2^{\mathrm{e}}$ éd., Francfort-BerneBerlin-Bruxelles-New York-Oxford-Vienne, 2000, pp. 129-131. - Commies, Nul ne s'y frotte, pp. 59-76. - ClÉMEnT, Antoine de Bourgogne, pp. 165-182. - Jeuris, Antoine Grand Bâtard de Bourgogne. Sur l'ensemble des bâtards ducaux bourguignons, consulter - pour l'essentiel, avec prudence : F. DE ReIFFenBerg, Enfants naturels du duc Philippe-le-Bon, dans Bulletins de l'Académie royale des Sciences, des Lettres et des Beaux-Arts de Belgique, t. 13, 1, 1846, pp. 172-187; t. 14, 1, 1847, pp. 585-597. - Bourgogne. Descendance illégitime de Jean sans Peur et de Philippe le Bon, dans Annuaire de la Noblesse de Belgique, t. 13, 1859, pp. 53-78. - M. Bergé, Les bâtards de la Maison de Bourgogne et leur descendance, dans L'Intermédiaire des Généalogistes, t. 60, 1955, pp. 316-408. - S. Gilloen, La vie et le statut des enfants illégitimes : l'exemple des bâtards de Philippe le Bon, Mém. de Maîtrise en Histoire, Université Charles-de-Gaulle-Lille 3, 1992. Nous n'avons pas pu consulter V. van Elewyck, Les bâtards de Philippe le Bon, duc de Bourgogne. Étude critique, 2 vol., Mém. de Lic. en Histoire, Université Libre de Bruxelles, 1988-1989. Il est également question de lui dans M. CARLIER, Kinderen van de minne? Bastaarden in het vifftiende-eeuwse Vlaanderen, Bruxelles, 2001, spéc. pp. 251-254. Enfin, plus généralement, voir les actes du colloque de Liège (16-17 oct. 2008) : La Bâtardise et l'exercice du pouvoir (XIII -début XVI siècle), éd. É. Bousmar, A. Marchandisse, B. Schnerb, Bruxelles, 2009, à paraître, dont plusieurs contributions sont consacrées à la Bourgogne ducale (B. SCHNERB, Des bâtards nobles au service du prince : l'exemple de la cour de Bourgogne (fin XIve-début Xve siècle). M. Maillard-Luypaert, Jean de Bourgogne, bâtard de Jean sans Peur, amant infidèle, père de famille nombreuse... et accessoirement évêque de Cambrai (1439-1479). - A. MARCHANDISSE, Corneille, Bâtard de Bourgogne. - J.-B. SANTAMARIA, Les bâtards à la Chambre des comptes de Lille : autour du cas de Denis de Pacy. - A. Duda, Les lettres de légitimation des ducs de Bourgogne. - C. Berry, La bâtardise au sein du lignage de Luxembourg. - S. SLANičKA, Le mécénat artistique comme stratégie de légitimation des bâtards princiers en France et en Italie. - L. НАвLот, L'emblématique des bâtards princiers à la fin du Moyen Âge, outil d'un nouveau pouvoir?).

9 Vaughan, Charles the Bold, p. 235, déclare que l'expression "Grand Bâtard » vient de Commynes et qu'il est connu de ses contemporains comme « le Bâtard de Bourgogne ».

10 Philippe de Commynes, Mémoires, éd. J. Blanchard, t. 1, Genève, 2007, p. 179.

11 JeAn De Roye, Journal connu sous le nom de chronique scandaleuse, éd. B. DE MANDRot, t. 2, Paris, 1896, pp. 43, 218.

12 Bernardino Corio, Storia di Milano, éd. A.-M. Guerra, t. 2, Turin, 1978, p. 1396 (Corio [*1459-† ap. 1503 \{1505?, 1513 ?\}], membre d'une famille proche et serviteur des familles ducales milanaises, est l'auteur de la première histoire de Milan en langue vulgaire, qui, bien que compilatoire, anecdotique et manquant de sens critique, constitue une source majeure : F. Petrucci, Art. Corio, Bernardino, dans DBI, t. 29, Rome, 1983, pp. 75-78. G. Chittolini, Art. Corio, Bernardino, dans LDM, éd. broch., t. 3, Munich, 2003, col. 236237 ; plus largement, voir S. Meschin, Uno storico umanista alla corte sforzesca. Biografia di Bernardino Corio, Milan, 1995). - Donato Bosso, Chronica, Milan, 1492, fol. 280 (ce Milanais noble et lettré ne fait guère preuve de réflexion, mais son œuvre a les avantages du témoignage direct: S. Peyronel, Art. Bossi, Donato, dans DBI, t. 13, Rome, 1971, pp. 298-299). - Ugo Caleffini, Croniche, 1471-1494, éd. F. Cazzola e. a., Ferrare, 2006, p. 113 (Caleffini [*c. 1439-† 1503], juriste, officier urbain et ducal, est notamment l'auteur 
George Chastelain et de Jean Molinet ${ }^{13}$, s'engage dans un périple de quelque cinq mois, une sorte de Grand Tour qui va le mener des États bourguignons à Naples et retour.

C'est une véritable escouade qui prend alors la route de l'Italie. En effet, plus d'une centaine de personnes ${ }^{14}$ accompagnent ou rejoignent le Bâtard, parfois après l'avoir quitté, au cours de cette expédition, lesquelles, pour partie, se dispersèrent en Italie afin de remplir quelque mission particulière ${ }^{15}$. Parmi celles-ci, outre une importante suite à cheval, l'on compte tout d'abord le premier fils qu'Antoine eut de son épouse Jeanne-Marie de La Viefville, Philippe de Bourgogne, seigneur de Beveren(-Waas) (*av. 1464-†1498), dont l'âge est alors compris entre onze et

d'un Diario, ici utilisé, qui est l'œuvre d'un témoin oculaire particulièrement attentif aux événements politico-militaires. T. BAcchI, Introduzione, dans UGo CALEFFInI, Croniche, pp. XV-XVII. - B. ANDREOlli e. a., Repertorio della cronachistica emiliano-romagnola [secc. IX-XV], Rome, 1991, pp. 199-203. - F. Petrucci, Art. Caleffini, Ugo, dans DBI, t. 16, Rome, 1973, pp. 647-650).

13 George Chastelain, Chronique, éd. J. B. M. C. Kervyn de Lettenhove, t. 5, Bruxelles, 1864, pp. 472, 481. - Jean Molinet, Chroniques, éd. G. Doutrepont et O. Jodogne, t. 1, Bruxelles, 1935, pp. 111, 164, 195, 462, 578.

14 Con circa cento cavali : Carteggi Milano Borgogna,t. 1, p. 407. - Dépêches des ambassadeurs milanais sur les campagnes de Charles le Hardi, duc de Bourgogne, de 1474 à 1477, éd. F. De Gingins la Sarra, t. 1, Paris-Genève, 1858, p. 47 ; cento cavalli: ASMi, Registri delle Missive, $\mathrm{n}^{\circ} 120$, fol. $96 \mathrm{v}^{\circ}, 26$ février 1475 ; so' in numero sua brigata ottanta cavali : Carteggi Milano Borgogna, t. 1, p. 410 ; au nombre denviron cent et dix personnes : Extraits des comptes des trésoriers généraux de Savoie, dans Chroniques de Yolande de France. Documents inédits, éd. L. MÉnABréA, Paris, 1859, p. 132, revu sur ASTo, Conti della Tesoreria ducale di Savoia, $\mathrm{n}^{\circ}$ 122, fol. $210 \mathrm{r}^{\circ}$; Summa cavalli CXV : Cicco [Francesco] Simonetta, I diari, éd. A. R. Natale, Archivio storico lombardo, 8e sér., t. 5, 1954-1955, année 81-82, 1956, p. 299 (sur cet important chroniqueur italien [* c. 1410-† 1480], membre éminent de l'entourage des Sforza, contemporain des événements ici évoqués, voir F. M. VAGLienti, Art. Simonetta. I. S. Cicco (Francesco), dans LDM, t. 7, col. 1921-1922 [et bibl.]. - M. N. CovinI, L'esercito del duca. Organizzazione militare et istituzioni al tempo degli Sforza (1450-1480), Rome, 1998, passim. - G. Lubkin, A Renaissance Court. Milan under Galeazzo Maria Sforza, Berkeley-Los Angeles-Londres, 1994, passim, un ouvrage de valeur auquel nous avons largement recouru) ; cum CL cavalli : ASMi, Archivio Sforzesco, Cartella 926, $1^{\text {er }}$ juillet 1475 (la mention concerne le mois de juillet 1475 : la suite avait eu tout loisir de s'étoffer depuis février-mars, comme semble l'indiquer ASMi, Archivio Sforzesco, Cartella 926, 22 juin 1475) ; cum docento cavalli: UGo CalefFini, Croniche, p. 113. La liste des participants nous est fournie par: Cicco Simonetta, Diari, pp. 298-299. - Carteggi Milano Borgogna, t. 1, pp. 409-410, 478-479. - Dépêches des ambassadeurs milanais, t. 1, pp. 106-109 (Der Briefwechsel Karls des Kühnen (1433-1477). Inventar, éd. W. PARAvicini e. a., t. 2, FrancfortBerlin-Berne-New York-Paris-Vienne, 1995, p. 295). - Extraits des comptes des trésoriers généraux de Savoie, p. 132.

15 Sources mentionnées en fin de n. précédente (Carteggi Milano Borgogna, Dépêches des ambassadeurs milanais, Briefwechsel). Ainsi Guillaume de Rochefort quittera Chambéry le 24 février 1475 pour rejoindre le Bâtard à Moncalieri (Carteggi Milano Borgogna, t. 1, p. 413.T. Zambarbieri, Milano e la Borgogna tra il 1474 e il 1477 : loro relazioni diplomatiche nel contesto dell'Europa mediana, dans Libri e Documenti, t. 8, 1, 1982, p. 52). 
vingt et un ans ${ }^{16}$. Le père et le fils sont accompagnés de Francesco d'Este (* 1429$\dagger$ ap. 20 juillet 1486) ${ }^{17}$, un autre bâtard, celui du duc de Ferrare Leonello d'Este, arrivé en 1444 à la cour de Philippe le Bon, où il partagera l'éducation du futur Téméraire et sera employé à la fois comme homme d'armes et comme diplomate, dans le cadre de dossiers italiens. À ce trio venaient s'ajouter Guillaume de Rochefort ${ }^{18}$, un Franc-Comtois, docteur in utroque, maître des requêtes ordinaires de l'hôtel et conseiller du duc jusqu'en 1476 puis momentanément de Marie de Bourgogne, lui aussi diplomate aguerri, spécialiste des questions italiennes, par ailleurs des plus appréciés à la cour de Savoie et de Milan, que Louis XI, coutumier du fait, parviendra à rallier à sa cause et créera chancelier de France (1483-1492),

16 Philippe († 1498), marié, en 1485, à Anne de Borselen, chevalier de la Toison d'or en 1478, conseiller-chambellan de Maximilien d'Autriche et de Philippe le Beau, dont il fut le témoin du mariage (1496), détint nombre de fonctions administratives et militaires dans le Namurois, en Artois et en Flandre, dont celles d'amiral des Pays-Bas (1488-1498) et de « chef de toutes les finances » (1495-1497). À son propos, voir J.-M. CAuchies, Art. Philippe de Bourgogne, seigneur de Beveren(-Waas), dans Les Chevaliers de l'Ordre de la Toison d'or, pp. 198-199, qui précise que, si sa date de naissance est inconnue, le mariage de ses parents eut lieu avant 1454 et son père lui céda la seigneurie de Beveren (Belgique, prov. Flandre orientale, arr. Sint-Niklaas, comm. Beveren) en 1464. - ID., Art. Philippe de Bourgogne, dans Nouvelle Biographie nationale, t. 3, Bruxelles, 1994, pp. 275-276.

17 WaLSH, Charles the Bold and Italy, p. 327 n. 32, pense que Francesco pourrait ne pas s'être rendu à Modène et à Ferrare durant le voyage de 1475. Si rien ne le prouve, en effet, le passage d'Antoine à Ferrare, bien attesté pour sa part (cf. infra), plaide plutôt dans le sens d'un séjour conjoint dans la capitale des Este. Sur Francesco d'Este, voir ibid., pp. 162, 164, 281-291, 361. - E. Kantorowicz, The Este Portrait by Roger van der Weyden, dans Journal of the Wartburg and Courtauld Institutes, t. 3, 1939-1940, reprod. anast., pp. 165 180. - A. Marchandisse, Le prince-évêque de Liège Jean de Heinsberg (1419-1455) : un modèle pour le Saint Georges de Pisanello ?, dans Annales de Bourgogne, t. 70, 1998, p. 140. Voyages d'ambassade en compagnie du Grand Bâtard en 1466 : http://ambassadeurs.plb.free. fr/ [accès limité]. Plus largement, sur Les étrangers à la cour de Bourgogne, voir le collectif du même titre, éd. B. SchNerB, Revue du Nord, t. 84, 2002, pp. 225-476.

18 Sur Guillaume de Rochefort(-sur-Nenon, France, dép. Jura, arr. Dole, ch.-1. de cant.) († 1492), voir J. Mangin, Guillaume de Rochefort conseiller de Charles le Téméraire et chancelier de France. Étude biographique suivie d'une notice sur Guy de Rochefort, dans Positions des Thèses de l'École des Chartes, 1936, pp. 117-121 (le dit né entre 1438 et 1440) (Nous n'avons pu retrouver la trace du mémoire en question.). - J. GAUTHIER, Note sur la véritable origine des deux chanceliers de France Guillaume (1483-1492) et Gui (1497-1508) de Rochefort, dans Bulletin historique et philologique du Comité des Travaux historiques et scientifiques, 1897, pp. 91-95. - J. Тнеияот, Dole. Genèse d'une capitale provinciale. Des origines à la fin du XVe siècle. Les structures et les hommes, t. 2, Dole, 1998, pp. 978-981 (le dit né vers 1438-1440) et passim. - M. HARSGOR, Recherches sur le personnel du conseil du roi sous Charles VIII et Louis XII, t. 2, Lille-Paris, 1980, pp. 1166 (le dit né entre 1435 et 1442)-1179. - J. Favier, Art. Rochefort (Guillaume de), dans Dictionnaire de la France médiévale, Paris, 1993, p. 825. - WALsh, Charles the Bold and Italy, pp. 27, 29, 66, 131, 157, 177-178, 231, 235, 242-243, 247, 285-286, 308, 345. - LouIs XI, Lettres, éd. J. VAESEN, t. 5, 1472-1475, Paris, 1895, p. 56 n. 2. - J. BARTIER, Légistes et gens de finances au XV siècle. Les conseillers des ducs de Bourgogne Philippe le Bon et Charles le Téméraire, t. 1, Bruxelles, 1955, pp. 281 n. 8, 284 n. 5, 418 n. 6. - E. Toutey, Charles le Téméraire et la Ligue de Constance, Paris, 1902, p. 193 n. 4. Deux ambassades en solitaire de Rochefort, en 1467 : http://ambassadeurs.plb.free.fr/ [accès limité]. 
un autre Comtois, parent de l'épouse du précédent, Louis Vurry ${ }^{19}$, issu d'une famille bourgeoise de Dole, alors doyen de la collégiale de la ville et membre du Parlement de Malines, et puis Jean de La Viefville ${ }^{20}$, chevalier, seigneur de Westrehem, conseiller et chambellan du duc, bailli de Saint-Quentin-en-Vermandois de 1462 à 1475 , cousin au quatrième degré de Jeanne de La Viefville, l'épouse d'Antoine. Étaient également présents Antoine d'Orlié21, membre d'une des plus vieilles familles savoyardes, vicaire de Cuneo de mai 1467 à mai 1469, gouverneur de Nice de 1469 à 1476, l'un des plus proches conseillers de Yolande de Savoie (* 1433$\dagger 1478)^{22}$, sœur du roi de France et veuve d'Amédée IX, futur bienheureux, qui

19 Fils de Gérard Vurry, diplomate et serviteur des ducs Philippe et Charles, cet universitaire fut doyen de Notre-Dame avant le 15 décembre 1474 et jusqu'à sa mort en janvier 1503, l'un des huit conseillers clercs siégeant au Parlement de Malines dès sa création en 1473, prédicateur d'indulgences dans les diocèses de Munster, Cologne et Utrecht entre 1484 et 1488, et maître des requêtes au Grand Conseil de Philippe le Beau en 1497. Guillaume de Rochefort (n. précéd.) épousa une Guye de Vurry ; son frère Guy, Catherine Vurry, la sœur de Louis. Voir Theurot, Dole, t. 2, pp. 1074, 1135. - Bartier, Légistes et gens de finances, t. 1, p. 419.

20 Jean de La Viefville, seigneur de Westrehem (France, dép. Pas-de-Calais, arr. Béthune, cant. Norrent-Fontes), fils de Guillaume, dit Lancelot de La Viefville, seigneur de Westrehem, et de Jeanne, dame de Bernemicourt. Lancelot était le fils de Sohier de La Viefville, seigneur de Thiennes et de Calonne-sur-la-Lys, lequel était le cousin germain de Pierre V de La Viefville, père de Jeanne de La Viefville, ce qui explique le cousinage au quatrième degré entre Jean et Jeanne. Jean de La Viefville a été conseiller et chambellan du duc. Voir ARRAS, Archives départementales du Pas-de-Calais, Rodière, ms. 86, I/2, p. 363 ; ms. 87, II/3, p. 5 ; ms. 91, IV/1, p. 137 ; ms. 236.; G. DuponT-FerRIER, Gallia regia ou État des officiers royaux des bailliages et des sénéchaussées de 1328 à 1515, t. 6, Paris, 1961, pp. 171-172, $\mathrm{n}^{\circ} 23428,23431$. - W. Paravicini, Guy de Brimeu. Der burgundische Staat und seine adlige Führungsschicht unter Karl dem Kühnen, Bonn, 1975, p. 264 n. 60.

21 Sur ce personnage, dont le nom apparaît sous des graphies diverses (Orly, Orlié, voire Orléans sic), accusé de cupidité effrénée et de malversations - il reçut en quantité fiefs, pensions et revenus divers du pouvoir ducal savoyard (ASTo, Materie politiche per rapporto all'interno, Protocolli ducali, $\mathrm{n}^{\circ}$ 118, fol. 288 ; Camerale Savoia, inv. 16, Conti della tesoreria generale di Savoia, $\mathrm{n}^{\circ} 117$, fol. $222 ; \mathrm{n}^{\circ} 121$, ff. $150 \mathrm{v}^{\circ}-151 \mathrm{r}^{\circ} ; \mathrm{n}^{\circ} 122$, fol. $319 \mathrm{v}^{\circ} ; \mathrm{n}^{\circ} 123$, ff. $313 \mathrm{v}^{\circ}$, $370 \mathrm{r}^{\mathrm{o}}, 404 \mathrm{r}^{\mathrm{o}}, 422 \mathrm{r}^{\mathrm{o}}$ ) -, † à la bataille de Morat, voir L. MARINI, Savoiardi e Piemontesi nello stato sabaudo (1418-1601), t. 1, 1418-1536, Rome, 1962, pp. 169, 185, 189, 195, 198, 199, 215, 221, 227, 236. - M. C. Daviso di Charvensod, La duchessa Iolanda, TurinMilan-Padoue-Florence-Rome-Naples-Catane-Palerme, 1935, pp. 32, 121, 131, 223.Haute magistrature de la ville et du comté de Nice. Gouverneurs de la ville et du comté de Nice, dans Nice historique, t. 6, 1904, p. 8. - Carteggi Milano Borgogna, t. 1, p. 478 n. 1.R. Brondy, B. Demotz, J.-P. Leguay, La Savoie de l'an mil à la Réforme, s. 1., 1984, p. 430. S. Guichenon, Histoire généalogique de la royale Maison de Savoie, t. 1, Lyon, 1660, p. 566. Cuneo (Italie, rég. Piémont, prov. Cuneo).

22 Sur Yolande, fille du roi de France Charles VII et de Marie d'Anjou, mariée en 1451 à Amédée IX de Savoie, voir B. Demotz, Art. Y. de France, dans LDM, t. 9, col. 415. - Daviso di Charvensod, La duchessa Iolanda.- Marini, Savoiardi e Piemontesi, t. 1, passim.E. Colombo, Iolanda, duchessa di Savoia (1465-1478). Studio storico, corredato di documenti inediti, dans Miscellanea di Storia italiana, t. 31, 1893, pp. 1-306. M. BROCARD, Yolande de France, duchesse de Savoie, sœur de Louis XI, Yens sur Morges, 1999, est un roman historique. 
exerce alors la régence au nom de son fils Philibert $\mathrm{I}^{\mathrm{er}}$, Rainieri Mancella ${ }^{23}$, un Napolitain, filleul du duc René d'Anjou et de Bar, comte de Provence, roi titulaire de Naples, écuyer de chambre du duc de Bourgogne et gouverneur de Nimègue, tout dévoué à la cause des Sforza de Milan, pro-angevin et donc parfaitement opposé aux Aragonais de Naples, ou encore le Piémontais Ameo ou Amedeo di Valperga $^{24}$, qui, en 1462, lorsque son père Giacomo, chancelier de Savoie et proche de Louis XI, fut assassiné afin de juguler l'influence française dans le duché, fuit ce dernier, et, en mai 1474, entra au service du duc de Bourgogne, d'abord comme écuyer d'écurie, puis, dès la fin de cette même année, au titre de commandant d'une compagnie de cent lances de l'armée ducale, avant de devenir gouverneur de Vaudémont. Gilles Gobet ${ }^{25}$, le Toison d'or du moment, roi d'armes de l'ordre entre 1468 et 1492 , participe également au voyage ${ }^{26}$.

23 Il était gouverneur de Nimègue et écuyer de chambre dès novembre 1473, et également gouverneur en 1482. On trouve la mention « René Mansel, filleul du roy et homme d'armes de la compaignie du conte de Campobas », 7 mai 1477, dans les comptes de René d'Anjou, cités par WaLsh, Charles the Bold and Italy, p. 187 n. 43. Sur ce personnage, voir Ibid., pp. 155, 161, 162, 168, 184 n. 5, 187 n. 43, 361. - Carteggi Milano Borgogna, t. 1, pp. 305 n. 8, 408. Paravicini, Guy de Brimeu, p. 336.

24 Ame(de)o était le fils de Jacopo/Giacomo di Valperga, comte de Masino, de noble famille piémontaise, chancelier du duc de Savoie de 1452 à 1456 avant d'être disgracié et de se trouver au centre de conflits entre la Savoie, d'une part, Milan et le dauphin Louis dont il est le protégé, le conseiller-chambellan et le chancelier à titre temporaire, d'autre part (R. AMBÜHL, Le séjour du futur Louis XI dans les pays de Philippe le Bon (1456-1461), Baisy-Thy, 2002, pp. 148-150. - G. Peyronnet, La politica italiana di Luigi Delfino di Francia (1444-1461), dans Rivista storica italiana, t. 64, 1952, pp. 39-44). Ame(de)o a participé à la bataille de Nancy (1477) et vivait toujours à la cour de France en 1491. Sur ce personnage, voir : Jean Molinet, Chroniques, t. 1, pp. 64-65. - Walsh, Charles the Bold and Italy, pp. 155, 157, 162, 184 n. 3, 162, 345, 351, 361, 365. Valperga (Italie, rég. Piémont, prov. Turin); Vaudémont (France, dép. Meurthe-et-Moselle, arr. Nancy, cant. Vézelise).

25 Gilles Gobet, dit « Fusil », auparavant appelé « Frise », devint poursuivant d'armes dès 1431 puis le successeur, en 1468, de Jean le Fèvre de Saint-Remy comme roi d'armes de la Toison d'or. F. DE GruBen, Les chapitres de la Toison d'or à l'époque bourguignonne (1430-1477), Louvain, 1997, pp. 37-38, 356-357 et passim. - F. Koller, Au service de la Toison d'or (Les officiers), Dison, 1971, p. 140.

26 Figurent également parmi cette suite, selon Cicco SimonetтA, Diari, pp. 298-299: Lo preposito de Sogni, cavalli IIII ; [...] Heuson Stoyvers, cavalli IIII ; Gullielmo de Hescusel, cavalli II ; Bourbon et George, cavalli VI; [...] lo Recipoyer et Deus capellanus, cavalli IIII ; Gullielmo, cavalli II ; les archies, cavalli VIII [... ]. Selon Carteggi Milano Borgogna, t. 1, p. 409, s'y ajoute misser Iohan Parmentreto dottore de lege. Tout comme les autres membres de cette ambassade, Salvatore de Clariciis, secrétaire de Charles le Téméraire, qui intervint à de très nombreuses reprises dans les relations diplomatiques entre ce dernier et Galeazzo Maria Sforza, est appelé à rejoindre le Bâtard à Milan à son retour de Naples : Ibid., t. 1, pp. 478-479. - Dépêches des ambassadeurs milanais, t. 1, pp. 106-109 [Ayme Valperga, Renero Macella e voy (et non noy comme indiqué dans ces deux éditions, vérifiées sur l'original, ASMi, Archivio Sforzesco, Potenze Estere, Cartella 516)]. Sur ce personnage, voir P. Cockshaw, Prosopographie des secrétaires de la cour de Bourgogne (1384-1477), Ostfildern, 2006, p. 84 (notice largement incomplète). - WALSH, Charles the Bold and Italy, passim. - Carteggi Milano Borgogna, t. 1-2, passim. - Dépêches des ambassadeurs milanais, t. 1, passim. 
Le lieu du départ de l'expédition semble avoir été $\mathrm{Neuss}^{27}$. En effet, si l'on en croit Molinet, Monseigneur le bastard de Bourgoigne vint pareillement audit siège, [...] puis, print congié à son frerre, le duc, et s'en alla au royaume de Naples ${ }^{28}$. Selon le roi Louis XI, dont les paroles sont rapportées par l'ambassadeur du duc de Milan à la cour de France, Cristoforo da Bollate ${ }^{29}$, le Bâtard a parcouru la Lorraine grâce à un sauf-conduit du duc René $\mathrm{II}^{30}$. La traversée du territoire lorrain fut des plus pénibles. C'est en tout cas ce que, selon toute vraisemblance aux environs du 18 février et à Genève, Antoine confie à Giovanni Pietro Panigarola ${ }^{31}$, qui se

27 Selon Vaughan, Charles the Bold, p. 236 et Walsh, Charles the Bold and Italy, p. 304, d'après LilLe, Archives départementales du Nord, B 2105, nº 67598, fol. $127 \mathrm{r}^{\circ}$, Antoine quitte Malines le 13 juillet 1475 (sic pour 1474) pour une ambassade auprès du roi d'Angleterre, du duc de Bretagne, des rois de Sicile, Portugal, Aragon, Naples, du pape et de Venise, voyage tous azimuts qui aurait été fragmenté et réorganisé, Antoine ayant gagné l'Angleterre, mais négligé les souverains ibériques. C'est d'ailleurs dans ce cadre que sera signée l'alliance militaire anglo-bourguignonne appelée "traité de Londres» du 25 juillet 1474 entre Édouard IV d'Angleterre et Charles de Bourgogne, représenté dans les négociations par son demi-frère Antoine (J.-M. CAuchies, Louis XI et Charles le Hardi. De Péronne à Nancy (1468-1477) : le conflit, Bruxelles, 1996, pp. 100, 103).

28 Jean Molinet, Chroniques, t. 1, p. 63. - KonRad Stolles, Thüringisch-Erfurtische Chronik, éd. L. F. Hesse, Bibliothek des litterarischen Vereins in Stuttgart, t. 32, 1854, p. 81.

29 Il remplit sa mission entre janvier 1473 et janvier 1475. À son propos, voir Dispatches with related Documents of Milanese Ambassadors in France, t. 3, 146611 march-29 june, éd. V. ILARDI, DeKalb, 1981, pp. XX-XXII.

30 Dépêches des ambassadeurs milanais, t. 1, p. 29. Les relations entre Charles le Téméraire et René II d'Anjou connurent une alternance de hauts et de bas. Le 15 octobre 1473, ils ratifient le traité de Nancy, lequel permet au premier de placer des garnisons dans certains châteaux du second. En décembre, ils visitent la Lorraine ensemble, tandis qu'au début de 1474, le cortège qui mène Philippe le Bon et Isabelle de Portugal vers leur dernière demeure, à Dijon, traverse les États de René. Mais, alors que le siège de Neuss commence, les troupes de Charles, qui transitent par la Lorraine, pillent la région comme s'ils traversaient un pays ennemi ; René décide alors de rallier la ligue comprenant Louis XI, l'empereur, les princes allemands, les villes d'Alsace et les cantons suisses ; il déclare secrètement qu'il renonce à l'accord qui le lie à Charles, avant de défier celui-ci le 9 mai 1475. Voir G. Poull, La Maison ducale de Lorraine devenue La Maison impériale et royale d'Autriche, de Hongrie et de Bohême, Nancy, 1991, p. 191. Cf., également, nos propos, supra, sur R. Mancella, dont il est par ailleurs dit qual alias fu col duca Zohanne (II de Lorraine, oncle de René II) (Carteggi Milano Borgogna, t. 1, p. 567).

31 Il est le premier ambassadeur résident, en France, exerçant ses fonctions du printemps 1465 à août 1468. Sa mission à la cour de Bourgogne s'étire de janvier 1475 à juillet 1476 : V. ILARDI, The First Permanent Embassy outside Italy: The Milanese Embassy at the French Court, 1464-1483, dans Politics, Religion and Diplomacy in Early Modern Europe. Essays in Honor of DeLamar Jensen, éd. M. R. Thorp, A. J. Slavin, Kirksville, 1994, pp. 6-9. - G. SoldiRondinin, Giovan Pietro Panigarola e il "reportage » moderno, dans Die Murtenschlacht. Ein Schweizer Ereignis in Europas Geschichte zwischen Mittelalter und Neuzeit, 1476-1976. Internationales Kolloquium zur 500-Jahr-Feier der Schlacht bei Murten, Murten 23.-25. April 1976. Kolloquiumsakten / La bataille de Morat. Un événement suisse d'histoire européenne entre le Moyen Âge et les Temps modernes, 1476-1976. Colloque international du $5^{e}$ centenaire de la bataille de Morat, Morat 23-25 avril 1976. Actes du colloque, Fribourg-Berne, 1976, pp. 135-154. - Dispatches, t. 3, pp. XV-XIX, L-LVIII. - F. Senatore, "Uno mundo de carta ». Forme e strutture della diplomazia sforzesca, Naples, 1998, passim. - Walsh, Charles the Bold and Italy, passim. 
rend à la cour de Bourgogne au titre d'ambassadeur du duc Sforza, ambassadeur qui profite de cette rencontre pour annoncer à un Bâtard réjoui la conclusion, le 30 janvier, du traité de Moncalieri, cette entente pleine de promesses entre les Bourguignons, Milan et la Savoie ${ }^{32}$. Le Bâtard se dirige ensuite vers les États savoyards, vers Moncalieri en l'occurrence, où il réside du 4 au 6 mars $^{33}$ : il lui a été servi à souper le dimanche soir - son hôte consentit à maintes dépenses en vins divers, légumes, fruits, confitures, poissons, céréales, huiles, sel, épices, bois et charbon afin que son séjour, dans divers hôtels, soit aussi agréable et paraisse aussi opulent que possible - et Antoine ast este deffroye [... ] luy et toute sa compagnie a toute outrance par la duchesse Yolande ${ }^{34}$. C'est le duc de Milan, Galeazzo Maria Sforza $(* 1444-1466-\dagger 1476)^{35}$ en l'espèce, qui sera le prochain hôte de l'équipée bourguignonne ${ }^{36}$.

Le séjour d'Antoine en terre milanaise, qu'encourageait le duc dès le 7 février $^{37}$, est plutôt bien documenté ${ }^{38}$. Le 26 février, Galeazzo, qui a appris quelque temps

32 Carteggi Milano Borgogna, t. 1, pp. 406-409 (le document est daté d'après le 18 et d'avant le 23 février 1475). - Dépêches des ambassadeurs milanais, t. 1, pp. 46-48 (le document, partiellement édité, est daté de «Besançon (?), fin de février 1475 (?). Date illisible »). Moncalieri (Italie, rég. Piémont, prov. Turin).

33 Comptes des trésoriers généraux, p. 132, qu'il convient de rectifier d'après le compte original (ASTo, Conti della Tesoreria ducale di Savoia, $\mathrm{n}^{\circ} 122$, fol. $210 \mathrm{r}^{\circ}$ : despuys le sabmedy IIII $^{e}$ jour du moys de mars lan mil IIII ${ }^{c}$ LXXV [...] jusque au lundy (et non jeudy) ensuyvant $V I^{e}$ jour dudit mois. Notons que le 18 février 1475, à Genève, Panigarola déclare qu'Antoine dicendo aveva aùto aviso che don Federico (Frédéric, fils de Ferrante d'Aragon, qui se rend à la cour de Bourgogne, voir infra) passava per Chiamberi e non per qui, e lui lo andaria a trovarlo, dove non staria che una nocte, et un giorno con Madama [...] (Carteggi Milano Borgogna, t. 1, p. 411).

34 ASTo, Conti della Tesoreria ducale di Savoia, $n^{\circ} 122$, ff. 210 r $^{\circ}-213 v^{0}, 286 v^{\circ}-287 r^{\circ}$. Pour cette étape du périple, nous avons consulté l'ensemble des sources narratives mentionnées par D. Chaubet, L'historiographie savoyarde, t. 1, Moyen Âge et Renaissance, s. 1., [1994], et, grâce au concours de Mme L. C. GentiLE, que nous remercions chaleureusement, nous avons pu prendre connaissance des sources inédites ad hoc à l'ASTo.

35 Sur Galeazzo Maria Sforza, voir Lubkin, A Renaissance Court. - C. SAntoro, Gli Sforza, rééd., Milan, 1994, pp. 111-173. - Storia di Milano, t. 7, L'età sforzesca dal 1450 al 1500, Milan, 1956, pp. 227-310. - G. Chiтtolini, Art. Sforza, dans LDM, t. 7, col. 1822 et 1824 (et bibl.).

36 Pour se rendre de Genève à Milan, Antoine a dû emprunter la via di Todeschi, c'est-à-dire passer par la Suisse, ce qui lui permettait d'atteindre Milan en cinq jours ; per Monsenise (Mont-Cenis, à la fois col et commune piémontaise de la province de Turin), il aurait fallu huit jours. Carteggi Milano Borgogna, t. 1, p. 411.

37 Ibid., t. 1, pp. 404-405.

38 Outre les chroniqueurs et les données éparses qu'ils fournissent, l'on mentionnera les Carteggi Milano Borgogna, les Dépêches des ambassadeurs milanais et les résultats de nos dépouillements à l'ASMi, Registri delle Missive, Registri ducali et Archivio Sforzesco, Potenze Estere, exploités, largement, par Lubkin, A Renaissance Court, pp. 211-212, 219220, partiellement, par ZAMBARBIERI, Milano e la Borgogna, pp. 33-69 et, de façon parfois peu précise, par WaLsh, Charles the Bold and Italy, passim. L'on y ajoutera Carteggio degli oratori mantovani alla corte sforzesca (1450-1500), t. 10, 1475-1477, éd. G. BAtTioni, Rome, 2008. 
auparavant que le Bâtard approchait du Milanais, adresse une rafale de questions à ses agents en Savoie, pays par lequel Antoine devait arriver ${ }^{39}$. Le 8 mars, il convie le Bâtard à venir le rejoindre à Mortara ${ }^{40}$. Accompagné de cavaliers, Tristano Sforza, seigneur de Castell'Arquato (* 1422/29-† 1477) ${ }^{41}$, qui est en quelque sorte l'alter ego d'Antoine, en l'occurrence le frère bâtard - légitimé - de Galeazzo, est chargé de prendre contact avec le Grand Bâtard à Verceil, du côté savoyard de la frontière, et de l'escorter durant l'ensemble de son déplacement dans les territoires Sforza $^{42}$. Le duc insiste par ailleurs pour que, en ces temps de Carême, le Bâtard se voie proposer du poisson au souper, poisson qui sera acheminé des lacs, en particulier du Lac Majeur ${ }^{43}$. Antoine et sa troupe pénètrent en territoire milanais et se rendent à Mortara le jeudi 9 mars puis à Vigevano le lendemain ${ }^{44}$. Si le duc s'est porté au devant d'eux, il ne descendra pas de cheval pour les accueillir, mais passera le week-end en leur compagnie et fera présent de deux chevaux et d'une mule à Antoine. C'est dans un bateau aux armes ducales que ce dernier fait son entrée à Milan, le 13 mars. Vient à sa rencontre un autre vaisseau, sur lequel ont prit place le Conseil privé, courtisans, officiers et surtout les fils du duc, Giangaleazzo Maria, comte de Pavie (*1469-1476-†1494), et Ermes Maria, marquis de Tortona $(* 1470-\uparrow 1503)^{45}$, respectivement âgés de 6 et 5 ans $^{46}$. Antoine sera logé

39 Tout spécialement à Antonio d'Appiano, ambassadeur milanais en Savoie. Cf. ASMi, Registri delle Missive, $\mathrm{n}^{\mathrm{o}} 117$, fol. $352 \mathrm{v}^{\mathrm{o}}-353 \mathrm{r}^{\mathrm{o}}, 26$ février $1475 ; \mathrm{n}^{\circ}$ 120, ff. $95 \mathrm{v}^{0}, 96 \mathrm{r}^{\mathrm{0}}-\mathrm{v}^{\mathrm{o}}, 26$ février 1475.

40 ASMi, Registri delle Missive, $\mathrm{n}^{\circ}$ 117, fol. $360 \mathrm{r}^{\circ}, 8$ mars 1475. - Carteggi Milano Borgogna, t. 1, p. 415. Voir aussi ASMi, Registri delle Missive, $\mathrm{n}^{\circ}$ 121, fol. $37 \mathrm{v}^{\mathrm{o}}, 8$ mars 1475. Mortara (Italie, rég. Lombardie, prov. Pavie).

41 Sa naissance est fixée à l'année 1422 selon A. Giulin, Di alcuni figli meno noti di Francesco I Sforza duca di Milano, dans Archivio storico lombardo, t. 43, 1916, p. 43, à l'année 1429 selon L. CERIONI, La diplomazia sforzesca nella seconda metà del Quatrocento e i suoi cifrari segreti, t. 1, Testo, Rome, 1970, p. 232. Ce conseiller ducal, condottiere réputé, légitimé en 1448, avait épousé Beatrice d'Este, fille et sœur naturelle des marquis Niccolò III, Leonello, Borso et Ercole I ${ }^{\text {er }}$ d'Este, et donc tante de Francesco (voir encore Santoro, Gli Sforza, p. 102 et W. K. Von Isenburg, Stammtafeln zur Geschichte der europäischen Staaten (Europäische Stammtafeln), t. 2, Marbourg, 1965, tabl. 123). Sur ce personnage, voir LuBkin, A Renaissance Court, passim. - Giulini, Di alcuni figli, pp. 43-46. - Cerioni, La diplomazia sforzesca, pp. 232-233. Castell'Arquato (Italie, rég. Émilie-Romagne, prov. Plaisance).

42 ASMi, Registri delle Missive, $n^{\circ}$ 120, ff. 95 v $^{\circ}, 98$ v $^{\circ}, 26$ et 28 février 1475; ASMi, Archivio Sforzesco, Cartella 855, 10 mars 1475. - Carteggi Milano Borgogna, t. 1, p. 415. - LuBKIN, A Renaissance Court, p. 211.

43 ASMi, Registri delle Missive, $\mathrm{n}^{0} 120$, fol. $100 \mathrm{v}^{\mathrm{o}}, 1^{\text {er }}$ mars 1475.

44 Galeazzo Maria Sforza prévoit un dîner à Mortara le 10 mars ; l'arrivée du Bâtard à Vigevano où réside le duc entre le 25 février et le 19 mars a donc dû s'effectuer dans la soirée du 10. ASMi, Registri delle Missive, $\mathrm{n}^{\circ}$ 121, fol. 40 v, 9 mars 1475. - Carteggi Milano Borgogna, t. 1, p. 415. - LubKIN, A Renaissance Court, p. 260.

45 Sur ces princes, voir Ibid. - Storia di Milano, t. 7, pp. 311-418. - G. Chittolini, Art. Sforza, dans $L D M$, t. 7, col. 1822 et 1824 (et bibl.). Vigevano (Italie, rég. Lombardie, prov. Pavie); Tortona (Italie, rég. Piémont, prov. Alexandrie).

46 Cicco Simonetta, Diari, p. 298. - Donato Bosso, Chronica, fol. 280. - Bernardino Corio, Storia di Milano, t. 2, p. 1396. -ASMi, Registri delle Missive, $\mathrm{n}^{\circ} 120$, ff. $111 \mathrm{r}^{\mathrm{o}}-112 \mathrm{r}^{\mathrm{o}}, 113 \mathrm{v}^{\mathrm{o}}$, $115 \mathrm{r}^{\mathrm{0}}-\mathrm{V}^{\mathrm{o}}, 10,11$ et 12 mars 1475. - Dépêches des ambassadeurs milanais, t. 1, p. 65. 
au Castello Sforzesco, dans les appartements occupés deux ans auparavant par un neveu du pape, Pietro Riario (†1474), cardinal-prêtre au titre de Saint-Sixte, et, pour l'heure, par les enfants ducaux, priés, pour la circonstance, de gagner le logis de leur mère, Bonne de Savoie, la belle-sœur de Yolande ${ }^{47}$. Durant son séjour milanais, les fils de Galeazzo vont, pour la première fois, jouer les hôtes attentifs pour visiteur de marque, réservant au Bâtard conversations affables, escortes à cheval et tour du propriétaire au Castello Sforzesco. Chaque jour, le comte de Pavie viendra converser avec un visiteur particulièrement impressionné par la courtoisie de l'enfant et amusé par le pas de deux, une moresque en l'occurrence, qu'entamera le petit marquis de Tortona avec une naine. Au matin du 16 mars ${ }^{48}$, le séjour du Bâtard touchait à sa fin. Les enfants ducaux, accompagnés du Conseil privé du duc, vinrent prendre congé d'Antoine et l'escortèrent jusqu'à la porte de la ville, ce dernier s'estimant particulièrement honoré par le lignage ducal, le Conseil et toute la cour milanaise ${ }^{49}$.

Antoine prit alors le chemin du royaume de Naples, destination ultime de son voyage. Accompagné de divers nobles napolitains venus à sa rencontre et accueilli non loin de Naples par Alfonso d'Aragon, duc de Calabre ${ }^{50}$, le fils aîné du roi Ferrante d'Aragon (* 1424-1458-† 1494) $)^{51}$, il pénétra dans la ville le 15 avril, vers vingt heures, y fut reçu en grande pompe par le souverain napolitain - un autre bâtard, en l'occurrence, celui du roi Alphonse V le Magnanime, légitimé en 1444 et logea dans la demeure de Diomède Caraffa, comte de Maddaloni, l'un des plus influents conseillers de Ferrante, après avoir été notamment le précepteur de ses fils $^{52}$. La présence à Naples de celui qui focalise l'attention de la cour tout entière sera l'occasion de festivités de grande ampleur, notamment de joutes. Le 20 avril 1475, le roi confirmait avoir été investi par Antoine de l'ordre de la Toison d'or et

47 ASMi, Registri delle Missive, n 120 , fol. 113 vº 11 mars 1475 ; Archivio Sforzesco, Cartella 926, 3 février (mars ?), 17 février et 8 mars, 11 et 12 mars 1475. - Dépêches des ambassadeurs milanais, t. 1, p. 65.

48 Carteggio degli oratori mantovani, t. 10, p. 115. - ASMi, Archivio Sforzesco, Cartella 926, 16 mars 1475.

49 ASMi, Archivio Sforzesco, Cartella 855, 13 mars 1475 ; Cartella 926, 15 et 16 mars 1475 ; Registri delle Missive, $\mathrm{n}^{\circ} 120$, ff. 127 ro $-128 \mathrm{r}^{\mathrm{o}}, 17$ mars 1475.

50 ASMi, Archivio Sforzesco, Potenze Estere, Cartella 227, 7 (le document annonce les faits) et 15 avril 1475. Sur ce personnage (* 1448-1494-† 1495), voir M. Del Treppo, Art. A. II., dans $L D M$, t. 1, col. 403-404.

51 Sur Ferrante, voir A. Ryder, Art. Ferdinando I (Ferrante) d'Aragona, dans DBI, t. 46, Rome, 1996, pp. 174-189 (et bibl.). - E. PontIERI, Per la storia del regno di Ferrante I d'Aragona re di Napoli. Studi e ricerche, 2e éd., s. 1., 1968.

52 La demeure de Caraffa († 1487) semble avoir fréquemment servi de demeure pour hôtes étrangers de marque, tel Sigismondo d'Este en 1473. Sur ce personnage, voir F. Petrucci, Art. Carafa, Diomede, dans DBI, t. 19, Rome, 1976, pp. 524-530 (et bibl.). Maddaloni (Italie, rég. Campanie, prov. Caserte). 
avoir juré de respecter les statuts ${ }^{53}$. L'on n'en sait guère plus, à l'heure actuelle, sur le séjour napolitain d'Antoine ${ }^{54}$, qui a dû s'achever au tout début mai ${ }^{55}$.

S'agissant de son voyage de retour, il ne manque pas de travaux, plus rarement de sources malheureusement, pour nous expliquer qu'il passa par Florence, par Ferrare ou par Venise.

Quoi qu'il en soit, dans un premier temps, Antoine gagna Rome et la cour du pape Sixte IV (1471-†1484). En ce printemps 1475, il vient grossir le nombre de ces souverains et princes étrangers, tels la reine de Danemark Dorothée de Brandenburg-Külmbach, Nicolas d'Ujlak, roi de Bosnie, ou encore Charlotte de Lusignan, reine de Chypre, qui défilent devant le souverain pontife, qu'ils soient, pour certains, venus en pèlerinage ou, pour la plupart, plaider pour que soit enfin concrétisée la croisade sans cesse programmée et abandonnée contre ces Turcs qui menaçaient leurs États ${ }^{56}$. Le 13 mai, dès l'annonce de l'arrivée du Bâtard, nombre de cardinaux, de nobles et de membres de la Maison du pape s'avancèrent vers lui et l'escortèrent jusqu'à Rome ${ }^{57}$. Le 16, il eut les honneurs de la table de Sixte IV, au Château Saint-Ange, ce qui était une première pour un prince séculier. Lors de la procession du Saint-Sacrement, le 25 mai, Antoine, en vêtements d'apparat, arborant des insignes, ornements et joyaux habituellement portés par le Saint-Père, se tint à la droite de ce dernier, de l'autre côté se trouvant le neveu de Sixte IV, Juliano della Rovere, cardinal-prêtre au titre de Saint-Pierre-aux-Liens, le futur Jules II, lequel, à Saint-Jean-de-Latran, multiplia les bénédictions à un Bâtard agenouillé devant

53 Giuliano Passero, Storie in forma di Giornali, éd. V. M. Altobelli, Naples, 1785, pp. 44, 45. - Notar Giacomo, Cronica di Napoli, éd. P. Garzilli, Naples, 1845, p. 129. - I diurnali del duca di Monteleone, éd. M. MANFredi, dans Rerum Italicarum Scriptores. Raccolta degli storici italiani dal cinquecento al millecinquecento ordinata da L. A. Muratori. Nuova edizione riveduta amplata e corretta, t. 21, 5, Bologne, 1958, p. 210. - ASMi, Archivio Sforzesco, Potenze Estere, Cartella 227, 15 et 28 avril 1475. - PonTiEri, Ferrante, p. 198. Pour l'étape napolitaine du parcours d'Antoine, nous avons consulté l'ensemble des sources narratives mentionnées par C. VECCE, Les chroniques napolitaines de la Renaissance, dans L'actualité et sa mise en écriture aux $X V^{e}-X V I^{e}$ et XVII siècles. Espagne, Italie France et Portugal. Études réunies par P. Civil et D. Boillet, Paris, 2005, pp. 84-85, une liste amplifiée et précisée par M. F. Senatore, que nous remercions chaleureusement.

54 L'on trouvait des traces des dépenses suscitées par le séjour d'Antoine dans ArChivio Di StATO Di NAPoli, Cedole di Tresoria, vol. 45, ff. 329 ro, 503 vo $, 504 \mathrm{r}^{\mathrm{o}}-\mathrm{v}^{\mathrm{o}}, 548 \mathrm{v}$ o $, 631 \mathrm{v}^{\mathrm{o}}, 632 \mathrm{v}^{\mathrm{o}}$, selon Pontieri, Ferrante, p. 198. Ces sources ont malheureusement été détruites au cours de la Seconde guerre mondiale.

55 ASMi, Archivio Sforzesco, Potenze Estere, Cartella 227, 1 ${ }^{\text {er }}$ mai 1475 ; Cartella 361, 28 mai 1475.

56 L. Pastor, Histoire des papes depuis la fin du Moyen Âge, 3e éd., t. 4, Paris, 1909, p. 259.

57 ASMi, Archivio Sforzesco, Potenze Estere, Cartella 79, 13 mai 1475. 
le pape. Celui-ci procéda alors à la légitimation et à l'anoblissement d'Antoine, faisant de lui le successeur de Charles, si ce dernier venait à mourir sans hoirs ${ }^{58}$.

Le 26 mai $^{59}$, le Bâtard laisse Rome pour Florence, où il arrive le 3 juin, loge dans les appartements pontificaux de Santa Maria Novella, et quitte la ville le $9^{60}$, après $\mathrm{y}$ avoir fait semble-t-il réaliser un médaillon à sa devise ${ }^{61}$. Il ne semble pas avoir été accueilli par les autorités de la Seigneurie. Celle-ci, à l'inverse d'émissaires florentins qui avaient tenté de le dissuader de visiter la ville, s'efforça, en vain, de lui faire rebrousser chemin au départ de Ferrare $^{62}$.

Après Florence, ce fut Bologne, puis Ferrare, précisément, le 15 juin, ce qui, à dire vrai, n'est pas une surprise puisque Antoine était accompagné du neveu du duc régnant, Ercole $\mathrm{I}^{\mathrm{er}} \mathrm{d}$ 'Este (* 1431-1471-1505). Ce dernier vint à la rencontre de son prestigieux hôte, à Torre Fossa, non loin de Ferrare, en compagnie de gentilshommes et de citadins, et le conduisit au palais Schifanoia, résidence d'été des Este et, pour la circonstance, du Bâtard et de sa suite. Le 16, Antoine visita le duc et son épouse Eleonora d'Aragon au château ducal, avant de souper en compagnie d'Ercole d'Este au palais Schifanoia. Le 17 en soirée, après le repas, Antoine fut invité par le duc à admirer ses différents bateaux, son bucintoro $^{63}$, ainsi que le Barcho, propriété de chasse nouvellement édifiée ${ }^{64}$.

58 De cronycke van Hollandt, Zeelandt en Vrieslant beghinnende van Adams tiden tot die geboerte ons heren Jhun Voertgaende tot den iare mcccc ende eerst begrepen en bewoent is gheweest van den Troyanen. En is inhoudende van die hertogen van Beueren Henegouwen en Bourgongen. Die tijt dat si ant graesscap geweest hebben. Met die cronike der biscoppen van uutrecht seer swerlic geectendeert en int lange bhaelt, s. 1. n. d. [Leyde, 1517], ff. 356 vo$357 \mathrm{r}^{\circ}$, reproduit dans Codex documentorum sacratissimarum indulgentiarum neerlandicarum. Verzameling van stukken betreffende de pauselijke aflaten in de Nederlanden (1300-1600), éd. P. FredericQ, La Haye, 1922, pp. 260-261. - Nicolaes Despars, Cronijcke van den lande ende graefscepe van Vlaenderen, éd. J. DE Jonghe, t. 4, Bruges, 1840, p. 102.

59 ASMi, Archivio Sforzesco, Potenze Estere, Cartella 79, 26 mai 1475.

60 Lionardo di Lorenzo Morelli, Cronaca, éd. I. De San Luigi, Delizie degli eruditi toscani, Florence, 1785, p. 191. Voir aussi Francesco Filarete et Angelo Manfidi, The Libro cerimoniale of the Florentine Republic, éd. R. C. TrexLer, Genève, 1978, p. 92 : E vassi perfino alle loro intrinsiche habitationi, verbigratia come fu al bastardo de Borgogna che fu ricevuto e fattegli le spese a Santa Maria Novella e simili, ecc.

61 J. FrIEDlander, Die italienischen schaumünzen des fünfzehnten jahrhunderts. 1430-1530. VII, dans Jahrbuch der königlich preussischen Kunstsammlungen, t. 2, 1881, p. 254.

62 ASMi, Archivio Sforzesco, Potenze Estere, Cartella 361, 9 juin 1475. Même si elles furent largement vaines, les recherches effectuées sur l'étape toscane du Bâtard l'auraient été encore bien plus sans l'aide précieuse de M. J. HAYEZ; nous lui en sommes très reconnaissants.

63 La visite aux «bucintori» ferrarais, vaisseaux non seulement de parade, mais aussi de voyage (T. Тиону, Herculean Ferrara. Ercole I d'Este, 1471-1515, and the Invention of a Ducal Capital, Cambridge, 1996, pp. 154-160), sera une étape fixe des voyageurs étrangers, jusqu'à Montaigne : cf. G. Ricci, I giovani, i morti. Sfide al Rinascimento, Bologne, 2007, pp. 117, 124.

64 Ugo Caleffini, Croniche, p. 113. Dès le 17 mars, selon Francesco d'Este, le passage par Ferrare était acquis (Carteggio degli oratori mantovani, t. 10, p. 115). Sur Ercole $\mathrm{I}^{\mathrm{er}} \mathrm{d}$ 'Este, duc de Ferrare, Modène et Reggio, voir T. DEAn, Art. Ercole I d'Este, dans DBI, t. 43, Rome, 
Conduits par deux gentilshommes vénitiens envoyés à cette fin par la Seigneurie ${ }^{65}$, le Bâtard et les siens se rendirent alors à Venise le 19 juin ; ils semblent y avoir résidé jusqu'au $24^{66}$. Il fut fait grand honneur à Antoine : le doge Pietro Mocenigo $(* c .1405-1474-\uparrow 1476)$ vint à sa rencontre dans son bucentaure ${ }^{67}$. Le Bâtard habitera la maison du duc de Ferrare, somptueusement décorée ${ }^{68}$. Il sera par la suite prié de visiter Mantoue par le marquis Lodovico Gonzaga (* 1414$1444-\uparrow 1478)^{69}$; le Bâtard se dit décidé à honorer l'invitation ${ }^{70}$. Arrivé à Brescia le

1993, pp. 97-107 (et bibl.). Pour l'étape ferraraise du périple d'Antoine, nous avons consulté l'ensemble des sources narratives mentionnées par ANDREOLLi, Repertorio, pp. 185-205, et bénéficié d'un soutien de valeur de la part de M. G. Ricci et de ses collaborateurs, ce en quoi nous les remercions chaleureusement.

65 Ugo Caleffini, Croniche, p. 113.

66 Date d'arrivée : «Lettre à "monseigneur de Pluvot", relative à Antoine, le grand bâtard de Bourgogne, Padoue, 26 juin 1475. Copie ou déchiffrement? », ainsi définie dans le Catalogue des manuscrits français, t. 4, Ancien fonds, 4587-5525, Paris, 1895, p. 482, et contenue dans le Recueil de pièces relatives principalement aux rapports de la France et de la Bourgogne sous les règnes de Charles VII et de Louis XI, 1447-1478, PARIs, Bibliothèque nationale de France, ms. fr. 5041, fol. 72 ro . - ASMi, Archivio Sforzesco, Potenze Estere, Cartella 361, 20 juin 1475. Date de départ: ASMi, Archivio Sforzesco, Potenze Estere, Cartella 361, 23 juin 1475.

67 Marino Sanudo, Vite dei dogi, Venise, Biblioteca nazionale Marciana, ms. it. VII, 801 (7152), parte III, fol. $7 \mathrm{v}^{\mathrm{o}}$. Selon la «Lettre à “monseigneur de Pluvot” » (n. précédente), le doge, indisposé, n'accueillit pas le Bâtard. Les recherches, tant dans les autres chroniques vénitiennes du temps que dans les archives (Cerimoniali et Notario du Collegio, Sénat, Commemoriali), se sont révélées infructueuses. Hormis celles relatives au ms. BnF, ces informations ont été collectées et nous ont été communiquées par M. P. Vuillemin et Mme É. Crouzet-Pavan, auxquels nous adressons de vifs remerciements. Sur Pietro Mocenigo, voir F. Sorelli, Art. Mocenigo, dans $L D M$, t. 6, col. 765 (et bibl.).

68 ASMi, Archivio Sforzesco, Potenze Estere, Cartella 361, 20 juin 1475.

69 C'est en tout cas l'information communiquée au duc de Milan, le 21 juin 1475 : Et chel Marchese de Mantua lha mandato ad pregare chel voglia fare la via da Mantua (ASMi, Archivio Sforzesco, Potenze Estere, Cartella 361). Dès le 17 mars 1475, l'ambassadeur du marquis de Mantoue à Milan pensait qu'Antoine allait passer par Mantoue à son retour de Naples (Carteggio degli oratori mantovani, t. 10, p. 115). Voir encore WALsH, Charles the Bold and Italy, p. 300. Sur les relations entre la Bourgogne et Mantoue, voir I. LAZZARINI, Mantoue et la Bourgogne au XV siècle, dans La cour de Bourgogne et l'Europe. Le rayonnement et les limites d'un modèle culturel. Actes du Colloque international, Paris, 9-11 octobre 2007, sous la dir. de W. Paravicini, sous presse. Nous remercions l'auteur de nous avoir transmis son étude avant parution et, tout comme M. G. BATTIONI, d'avoir accepté de partager avec nous ses connaissances sur Mantoue. Sur Ludovico Gonzaga, voir I. LazZarini, " Un bastione de mezo " : transformazioni istituzionali e dinemiche politiche, dans Storia di Mantova. Uomini, ambiente, economica, società, istituzioni, t. 1, éd. M. A. Romani, Mantoue, 2005, pp. 443505. - C. Mozzarelli, Mantova e i Gonzaga dal 1384 al 1707, Turin, 1987, pp. 23-36 et passim. - G. Coniglio, I Gonzaga, s. 1., 1967, pp. 52-65.

70 ASMi, Archivio Sforzesco, Potenze Estere, Cartella 361, 21 et 23 juin 1475. Le 27 juin, Antoine, qui semble proche de Crémone, mais dont l'on ne sait s'il s'est rendu ou non à Mantoue, semble continuer à occuper les pensées et les écrits du marquis : ASMi, Registri delle Missive, $\mathrm{n}^{\circ} 120$, fol. $335 \mathrm{v}^{\mathrm{o}}, 27$ juin 1475. 
29 juin, Antoine se rend ensuite à Malpaga, bastion de Bartolomeo Colleoni ${ }^{71}$, cette fois à la demande du fameux condottiere ${ }^{72}$, dont Charles le Téméraire sollicitera le concours à plusieurs reprises, en cette année 1475 comme auparavant ${ }^{73}$ - pourtant, Colleone était alors malade et proche de la mort -, et dont la rencontre avait peutêtre déjà motivé le passage d'Antoine par Venise. Malade lui aussi, celui-ci ne s'attarde pas à Malpaga, mais il se pourrait qu'il y ait reçu un message de son frère Charles lui décrivant la situation politique plus qu'alarmante que connaissaient alors ses États ${ }^{74}$.

Le 3 juillet $^{75}$, en soirée ${ }^{76}$, Antoine entame son second séjour à Milan, cette fois bien plus court. Ainsi que le duc, qui résidait alors à Pavie, l'avait de nouveau ordonné, sa cour et en particulier son frère bâtard lui réservèrent bon accueil et l'accompagnèrent à l'hôtel du Pozzo ${ }^{77}$. Il y profita de la compagnie de huit des plus honorables gentilshommes ducaux et fut comblé de victuailles, parmi lesquelles de grandes quantités de pain et de vin, trois roues de fromage vieux, quantité de

71 Sur ce personnage fameux (* c. 1400-† 1475), voir M. E. Mallett, Art. Colleoni, Bartolomeo, dans DBI, t. 27, Rome, 1982, pp. 9-19. - B. BеLотt, La vita di Bartolomeo Colleoni, Bergame, s. d. - M. E. Mallett, Venice and its Condottieri, 1404-1454, dans Renaissance Venice, éd. J. R. Hale, Londres, 1973, pp. 121-145. Voir encore, récemment, J. KoHL, Fama und Virtus. Bartolomeo Colleonis Grabkapelle, Berlin, 2004. Le château de Malpaga se trouve sur le territoire de Cavernago, une petite commune située en Lombardie, non loin de Bergame.

72 ASMi, Archivio Sforzesco, Potenze Estere, Cartella 361, 21 juin 1475.

73 Voir notamment ASMi, Archivio Sforzesco, Potenze Estere, Cartella 361, 8, 14 avril, 2 mai 1475. - W. PARAVIcini, Ein Spion in Malpaga, Zur Überlieferungsgeschichte der Urkunden des René d'Anjou und Karl des Kühnen für Bartolomeo Colleoni, dans Italia et Germania. Liber Amicorum Arnold Esch, éd. H. Keller, W. Paravicini, W. Schieder, Tübingen, 2001, pp. 469-487 (bibl. sur Colleoni). Cet auteur signale, dans WALsH, Charles the Bold and Italy, p. 464, qu'il prépare de nombreuses études sur les relations entre Charles et le condottiere.

74 ASMi, Archivio Sforzesco, Cartella 927, $1^{\text {er }}$ juillet 1475 (il logera au château de Cassano, forteresse proche de la frontière avec Venise). - Belott, Colleoni, p. 509.

75 Le $1^{\text {er }}$ juin 1475, Zaccaria Saggi, ambassadeur de Lodovico Gonzaga, marquis de Mantoue, à la cour de Milan, déclare el bastardo di Borgogna s'aspetta qui lunedi, soit le 5 juin. Alors qu'il devait être rentré pour mai, bien loin d'être à Milan à la date indiquée par Saggi, Antoine fera un détour qui l'occupera un mois. Carteggio degli oratori mantovani, t. 10, p. 144. Préparatifs de son second séjour en juin 1475 : ASMi, Archivio Sforzesco, Cartella 926, 8 juin 1475 ; Registri delle Missive, $n^{\circ} 120$, fol. $321 v^{\circ}, 21$ juin 1475. Le $1^{\text {er }}$ juillet, Antoine reçoit du duc un laisser-passer valable dans l'ensemble des États milanais (ASMi, Registri ducali, Cartella 109, Immunità, concessioni, cittadinanze, grazie, salvocondotti, patenti, fol. $594 \mathrm{v}^{\circ}$ ).

76 Ces informations viennent de l'ambassadeur mantouan (Carteggio degli oratori mantovani, t. 10, p. 166). Elles sont en contradiction avec celles avancées par Simonetta (Diari, p. 313), selon lequel l'arrivée d'Antoine a lieu le 2 juillet, vers 21 heures. Compte tenu du fait que la dépêche de l'ambassadeur mantouan est datée de Milan (4 juillet 1475) et que Simonetta, lui, le 2 juillet, est à Pavie, l'information proposée par le premier nous semble devoir être privilégiée.

77 [...] e l'ordine de allogiarlo a l'hostaria si serva fin qui, [...], selon Saggi, dans sa lettre du $1^{\text {er }}$ juin 1475. Carteggio degli oratori mantovani, t. 10, pp. 144, 166. Voir aussi ASMi, Archivio Sforzesco, Cartella 926, $1^{\text {er }}$ juillet 1475. 
volailles de types différents, dix-huit livres de massepain, des fruits, des noix, six pains de sucre fin, vingt-quatre flasques de malvoisie, le tout augmenté de cent vingt livres de cire $^{78}$. Tout cela ne va pas améliorer sa santé : on lui prête alors des maux d'estomac ${ }^{79}$. Bien que des plus généreux, Galeazzo ne souhaita pas rencontrer Antoine durant son second séjour ${ }^{80}$, alors que ce dernier, lui, multiplie les tentatives pour entrer en contact avec son hôte ${ }^{81}$. À dire vrai, le duc est courroucé. Il n'apprécie guère qu'à l'aller le Bâtard se soit présenté à Milan uniquement parce que le duché lombard était sur le chemin qui devait le mener dans le sud de la Péninsule. Lorsque, au retour, compte tenu de la signature préalable du traité de Moncalieri, Antoine est à présent chargé d'une ambassade auprès du duc de Milan, celui-ci ne la tient pas pour celle digne de ce nom qu'il s'estimait en droit d'attendre, une ambassade à laquelle il comptait répondre par l'envoi de son frère cadet, frère légitime cette fois, Ludovico, le futur Ludovico il Moro ${ }^{82}$. Et puis il y a les projets d'union matrimoniale entre la Bourgogne et Naples, qu'on lui présente comme abandonnés, mais dont il continue, semble-t-il, à soupçonner l'élaboration ${ }^{83}$. Un tel mépris à son égard amena Antoine à suspecter le duc de recevoir en secret des ambassadeurs français - Galeazzo Maria a beau s'en défendre, l'accusation pourrait bien ne pas être totalement dénuée de fondement ${ }^{84}$ - et à prétendre que ce dernier ne voulait pas lui accorder l'aide militaire promise au duc de Bourgogne ${ }^{85}$. Le 8 juillet, au terme de ce séjour roboratif à défaut d'être chaleureux, Antoine quitta Milan entouré de la brillante escorte habituelle, au sein de laquelle il découvrit une ancienne connaissance, fréquentée en Brabant, en l'occurrence l'évêque de Côme

78 Cicco Simonetta, Diari, p. 313. - ASMi, Archivio Sforzesco, Cartella 926, 22 juin 1475. Carteggio degli oratori mantovani, t. 10, p. 166.

79 Ibid., t. 10, pp. 168, 169.

80 [...] cosi di non volerlo vedere altramente. Ibid., t. 10, pp. 144, 166.

81 Notamment par le biais d'un de ses hérauts. Ibid., t. 10, pp. 167, 168.

82 ASMi, Archivio Sforzesco, Cartella 927, 2 et 8 juillet 1475.

83 Le duc n'explique pas autrement son ire le 9 juillet 1475 : Carteggi Milano Borgogna, t. 1, p. 565 .

84 Le 9 juillet 1475, il déclare : et delli ambaxatori francesi che debeno essere venuti da nuy secretamente dicemo non essere vero, [...] è stato qui da nuy alcuno ad nome del re di Franza, salvo Francesco Quirino, quale spazassemo in forma comuni, secundo te scrissemo alhora; altri non sono venuti, salvo uno nostro cremonese homo d'arme de Bofillo, quale mo' sono circa VIIJ zorni che zonse qui et vene de Roano dove se trovava el re de Franza (Ibid., t. 1, p. 566). L'on doit bien remarquer que, de ce nom Bofillo mentionné par Galeazzo Maria Sforza, est proche celui de Boffille de Juge, un familier de Louis XI, conseiller et chambellan, élevé à la dignité de vice-roi de Roussillon et de Cerdagne en juillet 1475. S'il ne s'agit pas de lui - il ne fut pas, semble-t-il, homme d'arme crémonais du duc de Milan - l'on peut penser à un parent. À son propos, voir F. PAsquier, Un favori de Louis XI, Boffille de Juge, comte de Castres, vice-roi de Roussillon, Albi, 1914. Voir encore ASMi, Archivio Sforzesco, Cartella 927,2 juillet 1475 .

85 P. A. Merkley, L. M. Merkley, Music and patronage in the Sforza court, Turnhout, 1999, p. 121. - Carteggio degli oratori mantovani, t. 10, p. 169 
(1466) et conseiller de Galeazzo Branda Castiglioni (* 1415-† 15 juillet 1487) ${ }^{86}$, auquel il ne manqua pas d'exprimer son amertume devant l'accueil pour le moins glacial que lui avait réservé le duc à son retour dans le Milanais ${ }^{87}$.

À présent, il était temps pour le Bâtard de regagner les États de son frère, lui qui, en février, espérait être de retour en Bourgogne pour le $1^{\mathrm{er}}$ mai et l'issue de la trêve conclue avec la France ${ }^{88}$. Antoine va donc se hâter, mais... « chi va piano, va sano e va lontano ». Le 10 juillet 1475, le revoilà à la cour de Yolande de Savoie, à Moncrivello, en Piémont, dans la résidence préférée de la régente, et il y prend manifestement du bon temps bien que son hôte ait été chargée par le duc Charles d'attirer son attention sur l'urgence de son retour en Bourgogne ${ }^{89}$. Il fut présenté à l'évêque de Turin, Jean de Compey (... 30 avril 1462-† 28 juin 1492) ${ }^{90}$,

86 Cet évêque de Côme (1466-1487), membre du Conseil privé du duc de Milan et volontiers employé comme ambassadeur par ce dernier, aurait été chanoine de la cathédrale de Liège (F. Petrucci, Art. Castiglioni, Branda, dans DBI, t. 22, Rome, 1979, pp. 126-129. - Cerioni, Diplomazia sforzesca, pp. 160-161. - Merkley, Merkley, Music and patronage in the Sforza court, pp. 80-81, 121). Seuls ses oncles Giovanni et Branda apparaissent dans J. DE THEUX DE Montuardin, Le chapitre de Saint-Lambert à Liège, t. 2, Bruxelles, 1871, pp. 189-190, 231-232 et É. PonCELET, Répertoire chronologique des conclusions capitulaires du chapitre cathédral à Liège. Supplément, dans Analectes pour servir à l'Histoire ecclésiastique de la Belgique, t. 23, 1892, p. 468. Peut-être l'appartenance du dernier mentionné au chapitre de Saint-Lambert permet-elle de ne pas nécessairement tenir pour une supercherie le fait que des bourses pour le collège de Pavie fondé en 1430 par Castiglione pouvaient aller à des étudiants désignés par le chapitre liégeois, même si le prélat lui-même remplaça Liège par Lisieux en 1437 (R. Herval, Trois grands évêques italiens en Normandie au XVe siècle : Branda, Zano et Giovanni Castiglione, dans Études normandes, t. 32, 1959, pp. 190-191).

87 Cicco Simonetta, Diari, p. 313. - ASMi, Archivio Sforzesco, Cartella 927, 7 (Antoine manifesta publiquement son mécontentement de diverses manières) et 8 juillet 1475 (le fils du Bâtard ne partira qu'un jour ou deux plus tard). - Carteggi Milano Borgogna, t. 1, pp. 565, 576. - Carteggio degli oratori mantovani, t. 10, p. 168. L'entourage du Bâtard a des avis contrastés sur la question de l'accueil d'Antoine lors de ce second séjour à Milan : Del non honorarlo tanto a questa volta como al'altra, dice domino Gulielmo (Guillaume de Rochefort) che vostra Excellentia fece tanto nel suo andre de là, che bene anchora per questo suo ritornare. Et lo governatore de Nizza (Antoine d'Orlié) disse cum una bona ciera ridendo : "Vuy non dite bene, domino Gulielmo. Io non dico che s'el signore duca gli fece bona ciera nel suo andare in là, ye la debbe fare megliore nel suo ritornare in qua, perchè tutto sarà honore et bruto di sua Signoria, che se portarà in quelle parte de là ». Carteggi Milano Borgogna, t. 1, pp. 557-558.

88 Ibid., t. 1, pp. 407-408. - Carteggio degli oratori mantovani, t. 10, p. 107.

89 J. Robert De Chevanne, Les guerres en Bourgogne de 1470 à 1475. Étude sur les interventions armées des Français au duché sous Charles le Téméraire, Paris, 1934, p. 287, qui cite un extrait d'un compte (1 ${ }^{\mathrm{er}}$ octobre 1474-30 septembre 1475) de Jean Vurry, receveur général de Bourgogne. Moncrivello (Italie, rég. Piémont, prov. Verceil).

90 Cet évêque de Turin (1468/9 [les deux titres mentionnés plus bas ne s'accordent pas sur le millésime]-1482), de Genève (1482-1484), puis archevêque de Tarentaise (1484-1492), notamment conseiller de la duchesse Yolande, partisan de Charles le Hardi en 1475, exerça les fonctions de chancelier de Savoie du 13 novembre 1462 au 7 mars 1464, après Giacomo Valperga, le père d'Ame(de)o. F.-C. Uginet, Art. Compey, Jean de, dans DBI, t. 27, Rome, 1982, pp. 689-692. - L. Binz, J. Emerr, C. SANTSCHI, Le diocèse de Genève. L'archidiocèse de Vienne en Dauphiné, Berne, 1980, pp. 106-107. 
venu à sa rencontre, puis accueilli à la porte du château par le duc de Savoie, Philibert, accompagné de ses deux frères, le futur duc Charles I $^{\text {er }}$ et Jacques Louis, respectivement âgés de dix, sept et cinq ans ${ }^{91}$, avant de rejoindre la duchesse dans sa chambre. Plus tard, il soupera en sa compagnie et dansera jusqu'à trois heures du matin, de sorte que, le lendemain, è stato in lecto fin hora de disnare ${ }^{92}$. Danse en galante compagnie - tutte le dame dela corte andarono ala camera del prefato Bastardo et li ballarono tutta nocte-, grasse matinée et collation de rôti et de malvoisie furent largement au programme des 11 et 12 juillet, avant, ce dernier jour, de déjeuner à Masino, résidence des Valperga, puis de poursuivre sa route par l'itinéraire le plus sûr ${ }^{93}$.

Il fit son entrée à Dijon le 22 juillet 1475. La Bourgogne était alors attaquée par des troupes françaises qui mettaient à profit un siège de Neuss à peine achevé et l'absence, en ces lieux, de l'élite militaire bourguignonne pour y déclencher des hostilités. Antoine prendra la tête d'une armée entre-temps privée d'Antoine de Luxembourg, son maréchal, capturé le 20 juin à Montreuillon, et parviendra à rétablir la situation. À l'automne, c'est une campagne victorieuse menée contre René II, duc de Lorraine et de Bar, qui l'attend, au départ de la Franche-Comté ${ }^{94}$.

Nous achevons ici notre évocation des diverses étapes du périple italien du Bâtard et des siens. Il importe désormais de nous interroger sur les mobiles de cette expédition.

Est-il besoin de le préciser : Antoine n'est pas l'instigateur de ce voyage. La tête pensante, bien sûr, c'est son frère et seigneur Charles, celui-là même qui, quelques mois plus tard, alors que Francesco d'Este, selon toute vraisemblance en plein accord avec le Bâtard Antoine, négociait quelque mariage burgondo-napolitain, déclara qu'il n'était au courant de rien, qu'il y était hostile - et pour cause, on le verra tout à l'heure - et qu'il était encore maître chez lui ${ }^{95}$. Manifestement, dans l'organisation de l'expédition de 1475, le duc de Bourgogne n'a rien laissé au hasard, en particulier quant à la composition de la délégation bourguignonne. Il y a tout d'abord le Bâtard lui-même, un homme manifestement très apprécié et tenu pour une personnalité éminente en Italie, choisi à dessein pour cette expédition

91 À leur sujet, voir B. Demotz, Art. K. I., dans LDM, t. 5, col. 993-994. - ID., Art. Ph. I., dans LDM, t. 6, col. 2055. - L. Usseglio, Bianca di Monferrato, duchessa di Savoia, Turin-Rome, 1892.

92 Carteggi Milano Borgogna, t. 1, pp. 566-569.

93 Ibid., t. 1, pp. 567, 575-576. Le château de Masino surplombe la ville de Caravino (Italie, rég. Piémont, prov. Turin).

94 A. Legual, Dijon et Louis XI. Notes sur quelques aspects de la Réunion de la Bourgogne, 1461-1483, II, Dijon et la guerre franco-bourguignonne 1470-1477, dans Annales de Bourgogne, t. 17, 1945, p. 111. - B. SCHNERB, L'État bourguignon, 1363-1477, Paris, 1999, pp. 422-423. - ID., "L'honneur de la maréchaussée 》. Maréchalat et maréchaux en Bourgogne des origines à la fin du XVe siècle, Turnhout, 2000, pp. 80-81.

95 Carteggi Milano Borgogna, t. 2, p. 93. ${ }^{-}$WaLsH, Charles the Bold and Italy, p. 286. 
italienne. Quant aux compagnons de voyage d'Antoine, l'on compte notamment parmi eux un diplomate de carrière, Guillaume de Rochefort, un très proche conseiller de la régente de Savoie, Antoine d'Orlié, un membre de la famille ducale de Ferrare, le bâtard d'Este, un fidèle serviteur de la maison Sforza, Ame(de)o di Valperga, et Rainieri Mancella, un Napolitain, certes honni par les Aragonais, mais un Napolitain tout de même, bref une équipe susceptible de faciliter les contacts en toute circonstance, quelles que soient les puissances italiennes rencontrées et visitées.

On l'a dit, l'expédition italienne du Bâtard trouve sa première raison d'être dans la gestion, en terre italienne, des cérémonies officielles au terme desquelles le roi Ferrante de Naples devait être pleinement investi de la Toison d'or. Un chapitre de l'ordre s'était ouvert à Valenciennes deux ans plus tôt, le $1^{\text {er }}$ mai 1473, en présence de l'ambassadeur du roi Ferrante, Francesco Bertini (* première moitié du XVe siècle-† 1475), évêque de Capaccio $^{96}$. Comme Charles souhaitait que des monarques ou de futurs monarques soient accueillis au sein de l'ordre, la question du choix des chevaliers fut débattue le 4 mai et, malgré le fait que Ferrante détenait déjà la Jarretière - en principe, les statuts de la Toison d'or interdisaient le cumul -, Ferrante est élu chevalier, au même titre que Ferdinand le Catholique, futur roi d'Aragon ${ }^{97}$. L'ordre charge alors Guy de Brimeu, seigneur d'Humbercourt, luimême devenu chevalier de la Toison d'or au cours du même chapitre, de se rendre auprès de l'heureux élu, accompagné de Toison d'or et du poursuivant d'armes Fusil $^{98}$. En août 1473, le duc Charles ordonne au greffier de l'ordre, Martin van Steenberghe ${ }^{99}$, de rédiger la patente confiant à Jean de Rubempré, seigneur de Biévène $^{100}$, lui aussi fait chevalier lors du chapitre de Valenciennes, la mission de faire parvenir à Ferrante, roi de Naples et de Sicile citra Farum, le collier de l'ordre; le 25 mai 1474, avant son investiture officielle en ses domaines, ainsi

96 De GruBen, Les chapitres de la Toison d'or, pp. 361-386. - Les Chevaliers de l'Ordre de la Toison d'or, pp. 164-183. Sur cet évêque d'Andria (1465-1471), puis de Capaccio (14711475), secrétaire et conseiller de Ferrante, ambassadeur napolitain près la cour de Charles le Téméraire dès les débuts du règne de ce dernier (P. M. Dover, Royal Diplomacy in Renaissance Italy : Ferrante d'Aragona (1458-1494) and his Ambassadors, dans Mediterranean Studies, t. 14, 2005, p. 87), qui joua un rôle dans le rapprochement du roi de Naples avec la coalition anti-française menée principalement par la Bourgogne, voir I. Walter, Art. Bertini, Francesco, dans $D B I$, t. 9, Rome, 1967, pp. 540-541.

97 De GruBEn, Les chapitres de la Toison d'or, pp. 372, 374.

98 Ibid., p. 378, selon laquelle le nouveau « Fusil»-Gobet jadis appelé « Fusil » était devenu roi d'armes de l'Ordre - était peut-être le héraut « Hainaut». Sur Guy de Brimeu, seigneur d'Humbercourt, bien connu, voir Paravicini, Guy de Brimeu. - P. De Win, Art. Guy de Brimeu, seigneur d'Humbercourt, comte de Megen, dans Les Chevaliers de l'Ordre de la Toison d'or, pp. $177-179$.

99 Sur ce greffier de l'Ordre de la Toison d'or de 1461 à 1491, voir De Gruben, Les chapitres de la Toison d'or, pp. 34-35, 297-298.

100 Sur ce proche du Téméraire, voir P. DE WIN, Art. Jean de Rubempré, seigneur de Biévène, dans Les Chevaliers de l'Ordre de la Toison d'or, pp. 171-173. - KOLLER, Au service de la Toison d'or, p. 114. 
que le permettaient les statuts de l'ordre, le roi confirmera avoir reçu le collier. Le $1^{\text {er }}$ décembre 1473, tout comme le chapitre de l'ordre avant lui, Charles charge Brimeu et van Steenberghe de se rendre en Campanie pour la confirmation de la désignation de Ferrante. L'on sait ce qu'il adviendra de ces dispositions : le 6 juillet 1474, le Bâtard sera substitué à Brimeu et investi de la mission qu'on lui connaît. Le 15 juillet, Antoine se voyait d'ailleurs confier un manteau de l'ordre, nécessaire à l'investiture du roi, par le trésorier Guillaume de Clugny ${ }^{101}$. Moins d'un an plus tard, Ferrante devenait chevalier de l'ordre de la Toison d'or au plein sens du terme ${ }^{102}$.

Loin de s'inscrire simplement dans l'inextricable lacis des cérémonies curiales, le port de la Toison d'or en terre napolitaine participe à n'en point douter du caractère éminemment politique du périple italien d'Antoine, qui prend place à une époque des plus troublées pour les États de Charles le Hardi. On le sait, en ces années 1474-1475, le duc cherche à s'imposer de force dans l'Empire et, pour ce faire, choisit de porter secours à son allié l'archevêque de Cologne Robert de Bavière, alors en butte aux attaques de ses sujets, soutenus par le landgrave Hermann de Hesse. Commence alors le siège de Neuss, où brûle le feu de la révolte ; il durera plus de dix mois, recouvre d'ailleurs complètement l'expédition d'Antoine et sera un parfait échec. Entre-temps, Charles s'était trouvé confronté à la Ligue de Constance, formée des villes de la vallée du Rhin, des confédérés suisses et de leurs alliés ; la Haute-Alsace, acquise en mort-gage par le duc en 1469, en avait profité pour se libérer de son emprise ; et le roi de France s'était allié à l'empereur Frédéric III (décembre 1474). Et le pire était à venir : dès mai 1475, la guerre contre la France reprend en Picardie comme en Bourgogne ; René II, duc de Lorraine et de Bar, adresse ses lettres de défi à Charles ; bientôt, l'allié sur lequel ce dernier compte tant, son beau-frère le roi Édouard IV d'Angleterre, va retourner sa veste et se vendre à Louis XI, le plus offrant. L'on comprend qu'en de telles circonstances, Charles ait ordonné le retour de son frère. L'on conçoit encore

101 Sur ce trésorier de l'Ordre de la Toison d'or de 1472 à 1477, voir De Gruben, Les chapitres de la Toison d'or, pp. 32-33, 372. - KolLer, Au service de la Toison d'or, pp. 63-64. Voir, tout récemment, la notice qui lui est consacrée, au titre d'évêque de Poitiers (1479-1481), dans L. VALLiĖRE, Fasti Ecclesiae Gallicanae. Répertoire prosopographique des évêques, dignitaires et chanoines de France de 1200 à 1500, t. 10, Diocèse de Poitiers, Turnhout, 2008, pp. 175-180.

102 J. Ruwet, Les archives et bibliothèques de Vienne et l'histoire de Belgique, Bruxelles, 1956, pp. 772-773, avec lequel, sur l'identité de celui à qui Rubempré est chargé de transmettre les insignes de l'Ordre, DE WIN, Art. Jean de Rubempré, est parfaitement en contradiction lorsqu'il dit que Rubempré « fut chargé le 14 août 1472 (sic pour 1473) d'apporter au fils de Jean II roi d'Aragon c'est-à-dire à Ferdinand, roi de Sicile (en l'occurrence Ferdinand le Catholique, roi de Sicile à partir de 1468, n. d. a.), tout juste élu chevalier, le collier et les insignes de l'Ordre de la Toison d'or; [...] le 25 mai 1474 le roi de Sicile accusa réception des insignes de l'Ordre ». Sur le parcours cérémoniel à l'issue duquel, traditionnellement, un souverain est fait chevalier de la Toison d'or, voir J. D. Boulton, The knights of the crown. The monarchical orders of Knighthood in later medieval Europe 1325-1520, Rochester, 2000, p. 376. 
mieux que, dans l'intervalle, il ait tenté de se ménager des alliés de poids dans la Péninsule italienne. Cette quête, qui visait en particulier à faire pièce à la menace que représentaient les Suisses, non seulement pour lui mais aussi pour ses alliés, Jacques de Savoie, comte de Romont, et la duchesse Yolande, passa par le traité de Moncalieri, l'on en a dit un mot auparavant, mais aussi, sans nul doute, par l'établissement de tout un ensemble de contacts, au cours du voyage d'Antoine, avec tout ce que l'Italie comptait de puissants, en particulier le roi de Naples et le pape $^{103}$.

Conférer la Toison d'or à Ferrante d'Aragon et se rendre à Naples pour recevoir officiellement le serment du roi, voilà qui constituait, pour Charles puis pour Antoine, à la fois une suite logique au traité de Saint-Omer de 1471, qui avait uni Bourgogne, Aragon et Naples contre le pouvoir français, et un excellent prétexte pour aller nouer sur place des relations politiques potentiellement fructueuses. Reste que cet ordre de chevalerie dont le roi de Naples allait être pleinement investi en avril 1475 ne fut pas loin d'anéantir dans l'œuf les accords signés en janvier avec Galeazzo Maria Sforza. Que l'on se mette un instant à sa place : il vient de s'allier à la Bourgogne et, pour lui montrer sa gratitude, Charles s'empresse de faire cadeau du prestigieux bélier... à un autre prince italien, prince que Galeazzo, qui plus est, ne tenait pas en haute estime ${ }^{104}$. Antoine lui-même semble avoir eu conscience du caractère pour le moins maladroit de la manœuvre ${ }^{105}$, lui qui, selon Panigarola, déclare que, si les accords de Moncalieri avaient été scellés plus tôt, il se serait bien passé de descendre à Naples et que l'octroi de la Toison à Ferrante était purement formel $^{106}$. À dire vrai, il est question depuis longtemps, dès le 18 mars 1475 à

103 SCHNERB, L'État bourguignon, pp. 414-424.

104 V. Ilard, Towards the tragedia d'Italia: Ferrante and Galeazzo Maria Sforza, friendly ennemies and hostile allies, dans The French Descent into Renaissance Italy 1494-95. Antecedents and Effects, éd. D. ABulafia, Aldershot, 1995, pp. 91-122.

105 Via des interlocuteurs susceptibles de rapporter ses paroles au duc, Antoine s'efforcera de rattraper la bourde. Ainsi est-t-il déclaré à G. M. Sforza, le 11 juillet $1475:[\ldots]$ el signore Bastardo cum tutta questa compagnia forono tanto honorati da vostra Excellentia nel suo andare in là, che non se porrìa dire più, et cento fiate è stata ricordata per camino et a Napoli quella bona ciera et honore facto per vostra Signoria, et che a Napoli nullo stava contento et non gli era facta tanta bona ciera da tutti insiema quanto era da madama vostra sorella duchessa de Calavria (Ippolita Maria Sforza [*1445-† 1488], cf. E. S. Welch, Between Milan and Naples: Ippolita Maria Sforza, duchess of Calabria, dans The French Descent, pp. 123-136. - F. M. Vaglienti, Art. Sforza Ippolita Maria, dans Dizionario biografico delle donne lombarde, 568-1968, éd. R. FARINA, Milan, 1995, pp. 1013-1015) sola, quale certamente haveva facto bona ciera et grande honore al prefato Bastardo, ritrovandosi hogi ad un giardino et domane ad un altro et cetera [...] (Carteggi Milano Borgogna, t. 1, p. 568).

106 Ibid., t. 1, pp. 408, 411 (col re Ferando sarà questo ordine bono per demonstratione, ma li effecti bisognarà siano col signore nostro et che si stringano insieme). - ZAMBARBIERI, Milano e la Borgogna, p. 52. 
tout le moins, de conférer le précieux sésame au Sforza ${ }^{107}$. Aussi, il est prévu de le lui remettre lors du chapitre qui doit se tenir en mai $1476^{108}$. Mais, le 12 juillet 1475, déjà, il est rapporté que le roi de Naples, qui avait appris que Galeazzo allait recevoir la Toison d'or, était prêt à restituer son propre collier, ne voulant en aucune manière siéger en compagnie de son rival milanais ${ }^{109}$.

La conclusion de mariages prometteurs était une autre façon, classique s'il en est, de nouer de solides alliances politiques ; elle pourrait avoir constitué une deuxième raison, pour Antoine, d'arpenter l'Italie. Et de fait, en cette matière aussi, il paraît très probable que le Bâtard a œuvré, assurément sur la base de dispositions prises avant son départ et qui changèrent en cours de route. Quelques années auparavant, Antoine s'était trouvé engagé dans des procédures matrimoniales le concernant très directement. En effet, en 1463, il avait été question de noces entre Philippe de Bourgogne, seigneur de Beveren, le fils du Bâtard, et la fille bâtarde du duc Francesco Sforza, Elisabetta Maria, future marquise de Montferrat (1456-1472), mais le dynaste milanais, on le constate, en décida autrement ${ }^{110}$. Au cours du voyage d'Antoine, alors que Ferrante de Naples souhaitait, par de séduisants mariages, se concilier le Bâtard et, à travers lui, le duc de Bourgogne, Francesco d'Este mènera des négociations en vue d'unions entre Antoine ou Philippe, son fils, et la fille du duc de Venosa, Francesco del Balzo, considéré comme le plus grand seigneur du royaume napolitain, par ailleurs apparenté au roi, ou du second avec l'une de celles du prince de Rossano, Marino Marzano, des négociations auxquelles ne sera réservée aucune suite favorable, notamment parce que le duc de Bourgogne s'y opposa ${ }^{111}$. Toutefois, durant le printemps 1475 , l'enjeu majeur du dispositif politique bourguignon, en matière de mariage, restait l'union que se devait de contracter sans tarder la fille et héritière unique de Charles le Téméraire, Marie. Née en 1457, et donc âgée de 18 ans en 1475, elle ne cessera d'être convoitée par tout ce que l'Europe des puissants comptait de jeunes princes alléchés par le véritable empire qui devait un jour ou l'autre revenir à la future duchesse. À l'époque qui nous intéresse, il a déjà été question de marier Marie à Ferdinand d'Aragon, à l'archiduc Maximilien, à Charles de France, le frère de Louis XI, au petit-fils de René ${ }^{\text {er }}$ d'Anjou, Nicolas, à Georges, duc de Clarence, le frère d'Édouard IV d'Angleterre, au futur roi de France Charles VIII, alors âgé

107 Carteggi Milano Borgogna, t. 1, p. 423. - ZAMBARBIERI, Milano e la Borgogna, p. 53. Il était déjà question de l'élection de Galeazzo Maria Sforza à l'Ordre de la Toison d'or en 1461 (Dispatches, t. 2, pp. 289, 371).

108 Il est ajouté que se interim la vostra Signoria ne vole segureza et promessa, la farà per scriptura, como fece al re Ferrando, la quale secundo l'ordine rimane depositata ne le mane de uno cavalero de l'ordine. Carteggi Milano Borgogna, t. 1, p. 540. Le chapitre de 1476 n'aura jamais lieu.

109 Carteggi Milano Borgogna, t. 1, p. 577.

110 Ibid., t. 1, pp. 176-179, 181-185.

111 Walsh, Charles the Bold and Italy, pp. 286-287. - ASMi, Archivio Sforzesco, Potenze Estere, Cartella 79, 26 mai 1475. 
d'un an et demi à peine, et à Philibert, le fils de Yolande de Savoie ${ }^{112}$. Au tout début de l'expédition italienne du Bâtard, une alliance avec Frédéric d'Aragon, le fils cadet de Ferrante de Naples, âgé d'une vingtaine d'années, envisagée depuis 1470, semble encore tenir la corde. Le 18 février 1475, alors qu'il est encore en Savoie et que, rappelons-le, il n'a aucune connaissance certaine de la signature du traité de Moncalieri, qui faisait du duc de Milan l'un des plus proches alliés de Charles de Bourgogne, Antoine semble faire le maximum pour pouvoir rencontrer Frédéric, alors en route pour la cour de Charles le Hardi ${ }^{113}$, prioritairement sans doute pour tenter de le convaincre de lui céder la main de sa fille ou tout au moins un commandement militaire qui lui donnera l'occasion de prouver qu'il pourrait faire un excellent duc de Bourgogne, accessoirement pour lui remettre l'Ordre napolitain de l'Hermine, en contrepartie de la Toison d'or concédée à son père ${ }^{114}$. Mais, en avril, le projet semble bien compromis : Humbercourt s'inquiète alors auprès de Panigarola de l'âge du comte de Pavie, le fils aîné du duc Sforza, en vue d'un possible mariage entre ce dernier et Marie de Bourgogne. À l'ambassadeur de Milan, qui lui fait remarquer que Giangaleazzo Maria est promis à la fille du roi de Naples, il répond, non sans cynisme, que ce dernier ne se montre guère parfait ami des Milanais et qu'il est habituel que les mariages soient décidés et annulés au gré des circonstances ${ }^{115}$. À la fin du voyage d'Antoine, les noces burgondo-napolitaines sont en passe d'avoir vécu ou, en tout cas, l'on en minimise la portée potentielle et l'on précise à qui veut bien l'entendre que, quoi qu'il arrive, tout cela n'aura aucune incidence sur l'estime que Charles porte au duc de Milan ${ }^{116}$. Federico finira d'ailleurs par obtenir, en septembre 1478, la main d'Anne de Savoie, la fille de Yolande et la nièce de Louis XI, sonnant ainsi l'un des glas de la politique italienne du Hardi ${ }^{117}$.

Dans la situation que connaissait le Téméraire en ces premiers mois de 1475, l'on peut à bon droit penser qu'il souhaitait voir son armée renforcée à la fois en hommes et en armes. On le sait, les ducs de Bourgogne ont régulièrement fait appel

112 B. SCHNERB, "La plus grande héritière du monde », dans Bruges à Beaune. Marie, l'héritage de Bourgogne, Beaune-Paris, s. d. [2000], pp. 24-28.

113 Carteggi Milano Borgogna, t. 1, pp. 410-411.

114 Ibid., p. 411. - Walsh, Charles the Bold and Italy, pp. 302 et sv. - Pontieri, Ferrante, pp. 163-208. - J. CALmetTE, Le projet de mariage bourguignon-napolitain en 1474 d'après une acquisition récente de la Bibliothèque nationale, dans Bibliothèque de l'École des Chartes, t. 72, 1911, pp. 459-472. - Boulton, The knights of the crown, p. 404.

115 Carteggi Milano Borgogna, t. 1, pp. 447-448. - ZAMBARBIERI, Milano e la Borgogna, p. 54.

116 Tel est par exemple le propos tenu pas Mancella à Antonio d'Appiano en juillet 1475 : Antonio, io no credo ch'el fosse may al mondo il magiore sforzesco ch'era il signore Bastardo et non credati che per questo parento havesse minuyto l'amore et affectione ch'el portava al signore duca vestro [...] (Carteggi Milano Borgogna, t. 1, p. 568). Il en va de même pour Antoine lui-même : ASMi, Archivio Sforzesco, Potenze Estere, Cartella 361, 21 juin 1475.

117 WaLsh, Charles the Bold and Italy, p. 322. 
à des hommes de guerre et de troupe italiens ${ }^{118}$. L'on se souviendra, par exemple, cas célèbre, de Castellain Vasc, ce capitaine lombard qui servit la Bourgogne, à la fois militairement et comme ambassadeur, au début du XV', et dont l'ascension fut telle qu'il finira par intégrer l'hôtel même du duc Jean sans Peur ${ }^{119}$. Deux générations plus tard, Castellain Vasc trouvera ses dignes successeurs en Cola di Monforte, comte de Campobasso, Jacques de Visque, comte de Saint-Martin, ou encore Troylo de Muro da Rossano, qui participera notamment, dès septembre 1475, à l'expédition lorraine dirigée par le Bâtard. Par ailleurs, jamais le recrutement de gens de guerre italiens, disciplinés, bien armés, dotés d'une infanterie puissante, ne se fera sur une aussi grande ampleur que sous le règne du Téméraire, qui, dans un premier temps, les engagea par contrat, selon des règles spécifiques, les considéra comme des troupes d'élite et les rémunéra davantage que les recrues de ses pays, avant de les intégrer au dispositif militaire bourguignon ordinaire ${ }^{120}$. Nous avons quelque raison de penser que, durant son déplacement en Italie, Antoine contribua au recrutement de soldats transalpins et se procura des armes. S'il faut en croire Louis XI, qui s'exprime au tout début février par le biais de Bollate, le Bâtard a seco tute gioie et thesoro tutale del Ducha de Brugogna per portarle in Italia ad impegnare per havere denari et gente ${ }^{121}$. Dès son arrivée à Milan, le 13 mars, Antoine déclare vouloir andare alla contrata delle arme, et [...] fare tore alcune mexure de arme $e^{122}$. De fait, à plusieurs reprises, le bâtard du duc de Milan Tristano Sforza conduira Antoine dans des boutiques d'armuriers, en particulier chez Antonio Missaglia, dont, douze années auparavant, Francesco Sforza avait recommandé la famille au duc de Bourgogne ${ }^{123}$. Il y commandera des armures pour lui-même,

118 De Boccace à 1494 et à la défaite de Fornoue, l'Italie vit, sur la lancée initiée par l'humaniste, dans l'idée d'une renaissance militaire, qui devait doubler la renaissance littéraire. Ce qui fait la valeur des Italiens, c'est leur vertu, opposée à la furor gauloise, topos qui court sur tout le Quattrocento. Si les Italiens sont certains de leur prédominance militaire, qui selon eux a glissé des Français aux Italiens, en partie à cause des échecs militaires français face aux Anglais, c'est aussi parce qu'elle se joint à une hégémonie culturelle et à l'excellence de leur modèle politique. Voir P. Gilli, Au miroir de l'humanisme, les représentants de la France dans la culture savante italienne à la fin du Moyen Âge (c. 1360-1490), Rome, 1997, pp. 483, $485,492$.

119 B. Schnerb, Un capitaine italien au service de Jean sans Peur : Castellain Vasc, dans Annales de Bourgogne, t. 64, 1992, pp. 5-38.

120 ID., Troylo da Rossano et les Italiens au service de Charles le Téméraire, dans Francia, t. 26, 1, 1999, pp. 103-128. - G. Soldi Rondinini, Condottieri italiens au service de Charles le Hardi, pendant les guerres de Suisse (1474-1477), dans Publication du Centre européen d'Études burgondo-médianes, t. 20, 1980, pp. 55-62.

121 Dépêches des ambassadeurs milanais, t. 1, p. 29.

122 Ibid., p. 65.

123 Carteggi Milano Borgogna, t. 1, pp. 187-188. - ASMi, Archivio Sforzesco, Cartella 926, 15 mars 1475 (Antoine paie per lettere de cambio, da quelli, che fano, per la compagnia delli Medici [la ponctuation est celle présente dans l'acte]). - M. MARTENS, La correspondance de caractère économique échangée par Francesco Sforza, duc de Milan, et Philippe le Bon, duc de Bourgogne (1450-1466), dans Bulletin de l'Institut historique belge de Rome, t. 27, 1952, p. 226. - C. GAIER, L'industrie et le commerce des armes dans les anciennes principautés belges du XIII à la fin du XV siècle, Paris, 1973, pp. 167 n. 165, 232 et n. 8, 274. 
ses fils et d'autres encore ${ }^{124}$, un matériel dont il connaissait à coup sûr toute la valeur, car, tant en Flandre, à Bruges par exemple, que dans la Comté, résidaient divers armuriers milanais, l'un d'eux s'intitulant d'ailleurs Armurier de Monsire le Bastart de Bourgogne ${ }^{125}$. En avril, Panigarola, qui le tient d'Humbercourt, précise que le Bâtard est porteur d'une requête de Charles, lequel souhaite que le duc de Milan, selon les engagements pris, lui adresse quatre cents lances ${ }^{126}$. En juin, et encore en juillet, le duc sollicitera l'envoi des mêmes hommes, de le quale havemo donate carcha al nostro caro et fedel cavalero, conseglier et primo chiamberla misser Antonio Bastardo de Burgogne, ce que, dans l'immédiat, le duc, attentif à l'évolution des relations entre France et Bourgogne, n'a nullement l'intention de faire ${ }^{127}$. Enfin, sur le chemin du retour, Antoine fera de nouveau ses emplettes d'armes à Milan ${ }^{128}$ et enrôlera quelques dizaines de soldats en pays savoyard ${ }^{129}$.

Une dernière raison a, selon nous, pu motiver le voyage du Bâtard : l'acte de légitimation posé par le pape Sixte IV. Qui est à l'origine de cette décision : Charles, Antoine, le souverain pontife ? Bien sûr, l'on ne sait trop. Il serait hasardeux de dire, par ailleurs, que, par cet acte, Antoine a souhaité se poser en rival de la jeune Marie. Reste que, en cette année 1475, rien ne prouve au duc Charles que sa succession ne connaîtra aucune difficulté. Louis XI veillera, durant l'aprèsNancy, à ce que soit tout le contraire. Marie, la fille unique de Charles, est jeune, célibataire et sans enfant. De frère et de sœur légitimes, ce dernier n'en a point. Alors oui, certes, selon le droit féodal bourguignon qui a présidé à la dévolution du duché de Bourgogne au duc Philippe le Hardi - et non celui des apanages, comme persistent à le dire tous ceux qui négligent les bons auteurs -, le duché pouvait

124 ASMi, Archivio Sforzesco, Cartella 926, 15 mars 1475.

125 WALsh, Relations between Milan and Burgundy, pp. 384-385. - GAIER, Industrie et commerce des armes, pp. 122-124, 274-275.

126 Carteggi Milano Borgogna, t. 1, p. 449.

127 Ibid., t. 1, p. 545 (citation). - Carteggio degli oratori mantovani, t. 10, p. 180. Le 9 mars 1475, le duc de Milan déclare à Charles le Hardi (et non au Bâtard Antoine, comme indiqué dans Carteggi Milano Borgogna, t. 1, p. 416, texte revu sur l'acte lui-même, conservé aux ASMi, Registri delle Missive, $\mathrm{n}^{\circ}$ 117, fol. $364 \mathrm{v}^{\circ}$ ) qu'il est prêt à affecter à son service Sforza Secondo, seigneur de Borgonuovo, son frère bâtard (P. SAvY, Un début dans la vie : Sforza Secondo jusqu'en 1467, dans Princes et princesses à la fin du Moyen Âge, éd. D. LetT et O. Mattéoni, Médiévales, t. 48, 2005, pp. 15-38; Cerioni, La diplomazia sforzesca, t. 1, p. 232). À la même date, ce dernier reçoit du duc l'autorisation de gagner l'armée bourguignonne (ASMi, Registri delle Missive, $\mathrm{n}^{\circ}$ 121, fol. $41 \mathrm{r}^{\circ}$ ). L'on ne sait si cette décision sera suivie d'effets (Walsh, Relations between Milan and Burgundy, pp. 382-383).

128 Lubkin, A Renaissance Court, p. 220.

129 Bruxelles, Archives générales du Royaume, Chambres des comptes, 25543, fol. $121 \mathrm{v}^{\circ}$. Antoine ne semble pas avoir profité de son voyage dans la Péninsule pour doter sa bibliothèque d'ouvrages italiens, enluminés ou non. C. VAn den Bergen-Pantens, Héraldique et bibliophilie : Le cas d'Antoine, Grand Bâtard de Bourgogne (1421-1504), dans Miscellanea Martin Wittek. Album de codicologie et de paléographie offert à Martin Wittek, éd. A. Raman, E. Manning, Louvain-Paris, 1993, pp. 323-334. 
connaître une transmission en ligne collatérale. Reste que, en 1475, hormis Agnès de Bourgogne, duchesse de Bourbon ${ }^{130}$, plus aucun des nombreux enfants de Jean sans Peur n'est encore en vie et, à défaut de Marie et de sa descendance, un Antoine légitimé devenait le meilleur des atouts pour l'avenir des pays bourguignons.

Durant son voyage d'Italie - et l'on conclura ainsi -, le Bâtard Antoine ne s'est pas ménagé, il n'a pas épargné sa salive. Il multiplia les visites, les contacts ; il se montra on ne peut plus agréable partout où il faisait étape et, en retour, manifestement, il fut, à une demi-exception près, accueilli avec les plus grands égards. Tout cela pour quoi, en fin de compte? Pour rien, ou presque. Quelles qu'aient pu être, au nom de son frère, les relations diplomatiques nouées par le Bâtard avec les souverains italiens - et l'on a vu que l'habileté et la finesse ne furent pas nécessairement au rendez-vous -, même si Antoine fut en mesure de recruter quelque soldatesque ou des armes, qu'il se soit agi soit de fomenter la plus foudroyante des coalitions contre la France, de l'étayer en faisant présent de la Toison d'or ou par la conclusion d'une union matrimoniale politiquement des plus profitables, soit de créer une force de frappe irrépressible, alors que le siège de Neuss se traînait lamentablement, Charles courait inexorablement au devant de Grandson, de Morat et de la débâcle de Nancy. Quant à Marie de Bourgogne, elle décédera sept ans plus tard. Alors que ses États échoient à un enfant, elle laisse, aux premiers comme au second, une situation politique somme toute aussi délicate que celle qui prévalait avant que, à la demande ou non, ensemble ou séparément, des frères de Bourgogne, Sixte IV n'offre à Antoine, en le légitimant, la capacité dynastique de régner.

130 Cette dernière meurt en 1476. A. Legual, Agnès de Bourgogne, duchesse de Bourbon (14051476), dans Les ducs de Bourbon, le Bourbonnais et le royaume de France à la fin du Moyen Âge, Yzeure, 2005, p. 160 (rééd. de l'art. paru dans Études bourbonnaises, $15^{\mathrm{e}}$ sér., nº 276, 1996, pp. 405-417). 
